\title{
LIDAR Metrology for Prescription Characterization and Alignment of Large Mirrors
}

\author{
B. Eegholm ${ }^{1}$ \\ Sigma Space Corp., Lanham, MD \\ W. Eichhorn \\ NASA Goddard Space Flight Center, Code 551, Greenbelt, MD \\ R. von Handorf \\ Ball Aerospace Corporation, Boulder, CO \\ J. Hayden \\ Sigma Space Corp., Lanham, MD \\ R. Ohl \\ NASA Goddard Space Flight Center, Code 551, Greenbelt, MD \\ G. Wenzel \\ Qinetic NA
}

\begin{abstract}
We describe the use of LIDAR, or "laser radar," (LR) as a fast, accurate, and non-contact tool for the measurement of the radius of curvature (RoC) of large mirrors. We report the results of a demonstration of this concept using a commercial laser radar system. We measured the RoC of a $1.4 \mathrm{~m} \times 1 \mathrm{~m}$ spherical mirror with a nominal RoC of $4.6 \mathrm{~m}$ with a manufacturing tolerance of $4600 \mathrm{~mm}+/-6 \mathrm{~mm}$. The prescription of the mirror is related to its role as ground support equipment used in the test of part of the James Webb Space Telescope (JWST). The RoC of such a large mirror is not easily measured without contacting the surface. From a position near the center of curvature of the mirror, the LIDAR scanned the mirror surface, sampling it with 1 point per $3.5 \mathrm{~cm}^{2}$. The measurement consisted of 3983 points and lasted only a few minutes. The laser radar uses the LIDAR signal to provide range, and encoder information from angular azimuth and elevation rotation stages provide the spherical coordinates of a given point. A best-fit to a sphere of the measured points was performed. The resulting RoC was within $20 \mathrm{ppm}$ of the nominal RoC, also showing good agreement with the results of a laser tracker-based, contact metrology. This paper also discusses parameters such as test alignment, scan density, and optical surface type, as well as future possible application for full prescription characterization of aspherical mirrors, including radius, conic, off-axis distance, and aperture.
\end{abstract}

\footnotetext{
${ }^{1}$ Bente.H.Eegholm@nasa.gov, tel. 301286 4342, fax 301286 0204, www.nasa.gov
} 
The trend among missions developing space telescopes, terrestrial telescopes, and other instruments for astrophysical research is toward systems with larger entrance apertures; i.e., larger, concave, primary mirrors. The driver behind this is to meet demanding sensitivity and angular resolution requirements for science missions. On the other hand, controlling and improving cost per unit area or other cost metric is also important, which is sought achieved with the improvements to the mirror fabrication process and later steps of telescope construction. However, optical testing is an integral part of the mirror fabrication and verification process and, despite their ubiquity, testing of aspheric mirrors remains a demanding task. In this paper, we show that, for both spherical and aspheric mirrors, use of laser radar (LR) technology can serve as a valuable process improvement for the fabrication and test phases of large optics. Previous work has described how the LR is a candidate for versatile, non-contact, multi-purpose metrology systems that can measure large structures, which consist of different materials, while minimizing cost, manpower, time, data acquisition, data analysis, etc. [1]. In this work, we extend the study to include the direct measurement of the optical surface of large, concave mirrors.

Currently, various Coordinate Measurement Machine (CMM) -like measurement systems are used as process feedback during the mirror blank fabrication process (e.g., grinding). During finer polishing steps, CMM-like metrology systems and interferometry or other optical tests are used to help complete fabrication, including final determination of the mirror prescription (e.g., radius, conic, aperture location and size) and surface figure error (i.e., departure from some reference shape). During mirror assembly to a telescope structure, various large volume measurement techniques are used as feedback to align the optic, such as systems based on portable CMMs, laser trackers, or theodolites, while interferometry or other wavefront-sensing is used to verify the optical performance with respect to some standard.[2, 3] The in-process and final measurements of mirror prescription and alignment often involve direct, physical contact with some portion of the mirrors optical surface, which can result in some damage and/or contamination risk to the flight optic.[2]

The commercial LR emits its Class II $1550 \mathrm{~nm}$ measurement beam vertically from the head of the instrument through a $\sim 40 \mathrm{~mm}$ circular exit aperture, which contains an optical train capable of focusing the measurement beam on measurement objects at distances between $\sim 1 \mathrm{~m}$ and $24 \mathrm{~m} \mathrm{[4].}{ }^{2}$ A flat mirror folds the measurement beam and points it to the object under test via azimuth and elevation stages. The fold mirror is positioned about $100 \mathrm{~mm}$ from the exit aperture. The azimuth stage rotates for $>360$ degree coverage horizontally, while the elevation stage provides about \pm 45 degree vertical pointing about the horizon.

The LR's optical train produces a point spread function on the test surface it is focused on of roughly diffraction limited size, corresponding to the limiting $40 \mathrm{~mm}$ circular aperture of the transmitter and the appropriate distance to the surface. For a distance of $4600 \mathrm{~mm}$, the spot size is $\sim 0.217 \mathrm{~mm}$.

The uncertainty in the absolute range measurement is in practice $\sim 15 \mu \mathrm{m}$ (1-sigma), limited by environmental effects, calibration, and instrument noise. Driven by the angular error associated with the rotation stages, the absolute, linear distance uncertainty in axes approximately orthogonal to the range direction is $\sim 10 \mu \mathrm{m}$ per $1 \mathrm{~m}$ of range (1-sigma). In a stable measurement environment with generous test time, the aforementioned uncertainties can be improved with multiple measurements and additional averaging, as well as by making measurements from various vantage points, or instrument "stations," and combining the data via further averaging or a bundling-type algorithm. ${ }^{5}$ We use Spatial Analyzer ${ }^{\mathrm{TM}}$ (SA) from New River Kinematics, Inc., Williamsburg, Va., www.kinematics.com to control the LR and perform data reduction and most analysis tasks.

\footnotetext{
${ }^{2}$ This range is relevant for the Nikon MV224 unit, www.nikonmetrology.com.
} 
The LR can operate in a number of different pre-set or user-defined modes. Grid-like scans of various precision are commonly used to measure mechanical surfaces. The centroid of mechanical holes for pins or fasteners can be found via another scan-like measurement algorithm. A scanning search algorithm is used to find the virtual center of convex spheres, such as "tooling balls." The LR is a coherent, frequency modulated instrument which detects the return signal from the target and optically mixes it with an internal reference signal to obtain the time of flight to the target. Multiple measurements made in quick succession are made and averaged to improve the precision. Data from a weather station is used in the conversion of the time of flight measurement to range. The LR is sensitive to a large range of return signals, enabling measurement of many different surface and target types, including both matt-finish, mechanical surfaces that produce faint scatter returns and mirror-like, specular surfaces that produce bright returns that follow the law of reflection.

\section{MEASUREMENT OF MIRROR RADIUS OF CURVATURE USING THE LASER RADAR}

As a demonstration of the capability of the LR to measure radius of curvature, we measured the radius of curvature of a spherical, high quality mirror with a nominal radius of 4600mm (Figure 1) [5]. The mirror has a rectangular physical aperture of $1 \mathrm{~m}$ by $1.4 \mathrm{~m}$, which corresponds to a circular physical aperture mirror with a diameter of $1.7 \mathrm{~m}$. The rectangular aperture of the mirror is related to its role as ground support equipment used in the verification of part of NASA's James Webb Space Telescope [6].

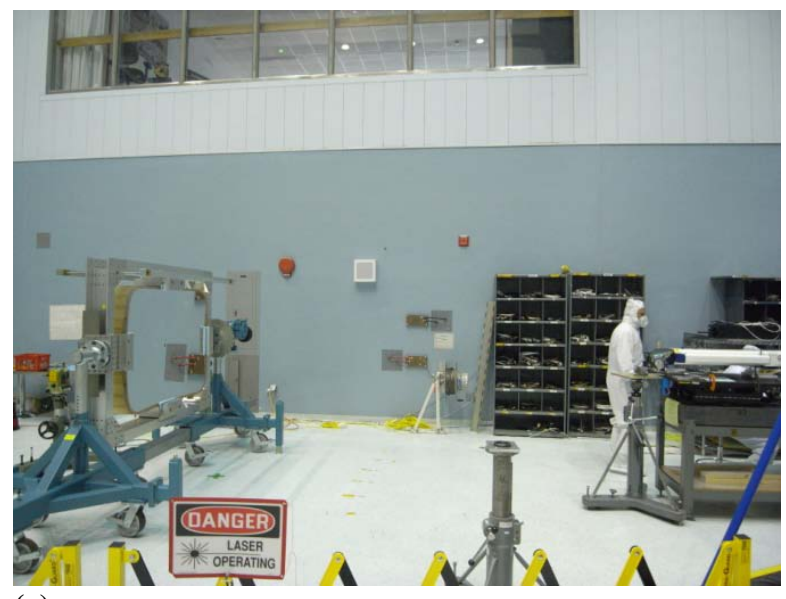

(a)

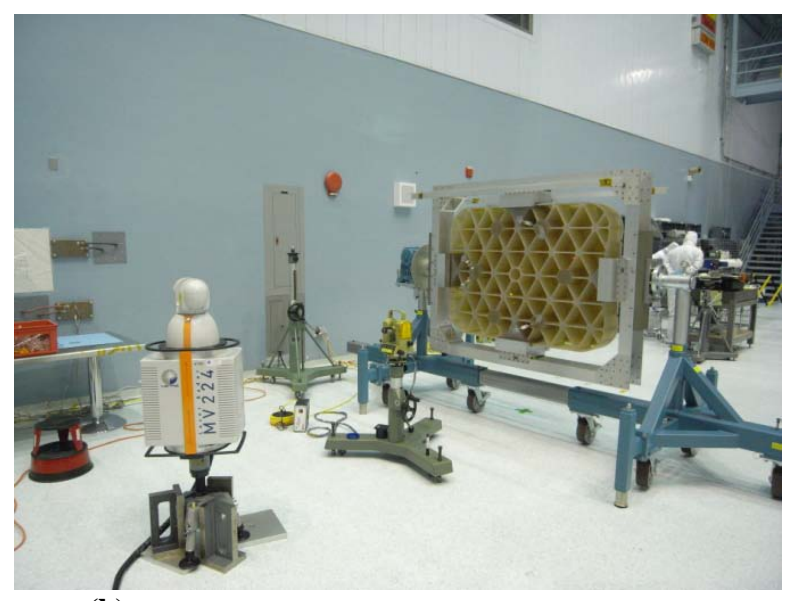

(b)

Figure 1.

a. Photograph showing the $\sim 1.7 \mathrm{~m}$ diameter spherical mirror (left) and the (4D 4020HP) interferometer (right) during figure error testing.

b. Photograph showing the LR MV224 (left), theodolite (center), and the mirror (right) during measurement of optomechanical alignment fiducials on the rear surface of the mirror substrate.

The mirror was held vertical in a dedicated mount. From a position near the center of curvature (CoC), the LR was focused to a point close to the mirror surface. The LR performed a metrology scan on the mirror surface, sampling it with a density of $\sim 1$ point per $3.5 \mathrm{~cm}^{2}$. The encoders move the laser radar head in the vertical direction, then in the horizontal direction during a scan. The LR scans the surface from top to bottom, for this measurement set to acquire a data point every $12.7 \mathrm{~mm}$ in the vertical direction (point spacing). When reaching the boundary defining the lower measurable edge of the mirror, the LR head moves back to the upper edge of the mirror, and horizontally $25.4 \mathrm{~mm}$ (line spacing) to acquire the next vertical line of points. 
Before staring the scan, the boundaries for the scan are defined. 8 tooling balls are placed around the mirror perimeter, 2 on each side. When defining the scan, the coordinates of the leftmost, rightmost, top and bottom points are defined to lie well within the physical edge of the mirror, in order to avoid the bevel of the mirror to affect the measurement of the radius of curvature (Figure 2).

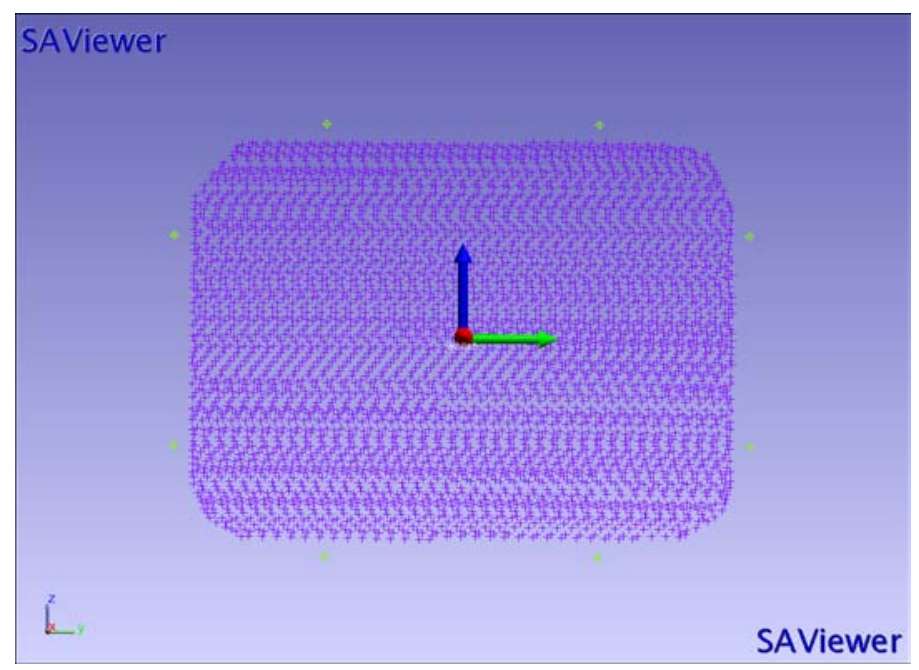

Figure 2. The scan points (blue) with density 1 point per $3.5 \mathrm{~cm}^{2}$ and the tooling ball points (green) defining the outer boundary of the scan.

The LR was positioned close to the center of curvature of the mirror. An interferometer equipped with a diverging lens, allowed interferometric measurement of the figure error of the full mirror. The diverger lens was focused at a point $19 \mathrm{~mm}$ outside the interferometer aperture as seen in Figure 3. A tooling ball was positioned at that focal point, and was used to indicate the position of the LR.

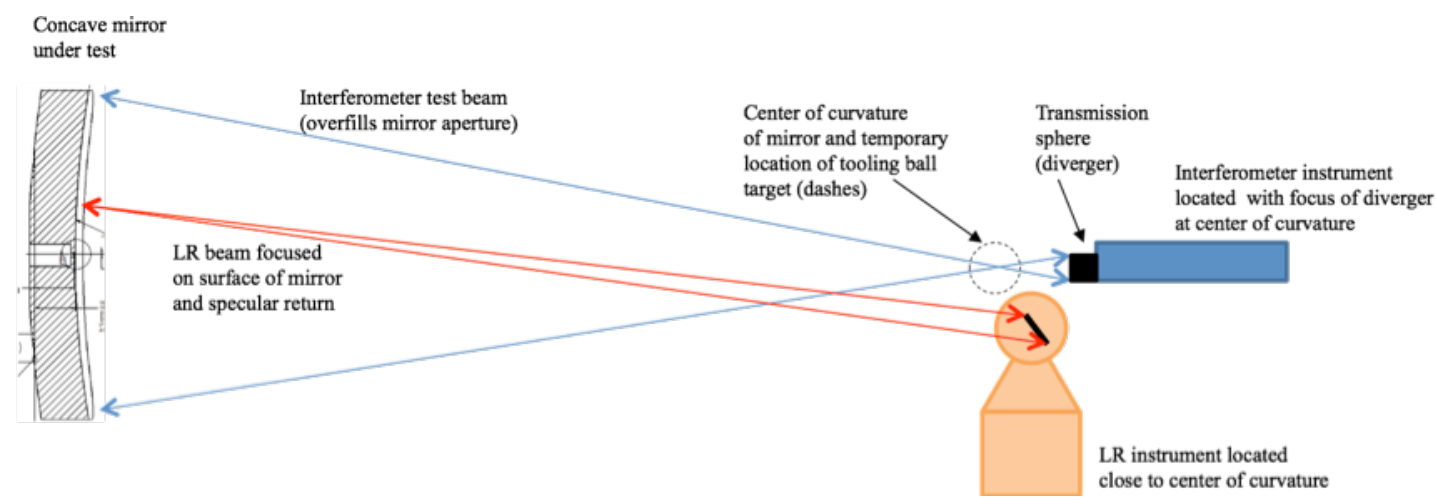

Figure 3. Schematic showing a large mirror under test using laser radar (LR) and interferometry. The mirror to the left measured by an interferometer with a diverging lens which focuses $19 \mathrm{~mm}$ in front of the interferometer aperture. The LR measures the prescription of the mirror (i.e., radius of curvature, conic constant, aperture size and location) and aids in alignment of the interferometer to the mirror. 
After the interferometric test, a high precision tooling ball with a NIST-traceable calibration was placed in the focus position of the diverger lens. When the diverging beam covers the full mirror aperture, this position represents the CoC. The tooling ball position was then used to position the LR close to the CoC.

The full area scan of the mirror contained 3983 points with a density of 1 point per $3.5 \mathrm{~cm}^{2}$. This dataset was run through a least-squares fit to a sphere available in the Spatial Analyzer software [7]. The tolerance for the points was set to $0.012 \mathrm{~mm}$, and points exceeding this were eliminated from the fit. Figure 4 shows the distribution of points. The resulting radius of curvature of the mirror was 4600.075 $\mathrm{mm}$, with a rms of $0.005 \mathrm{~mm}$. This is an excellent match to the manufacturing requirement, which - rather loosely - was 4594-4606mm.

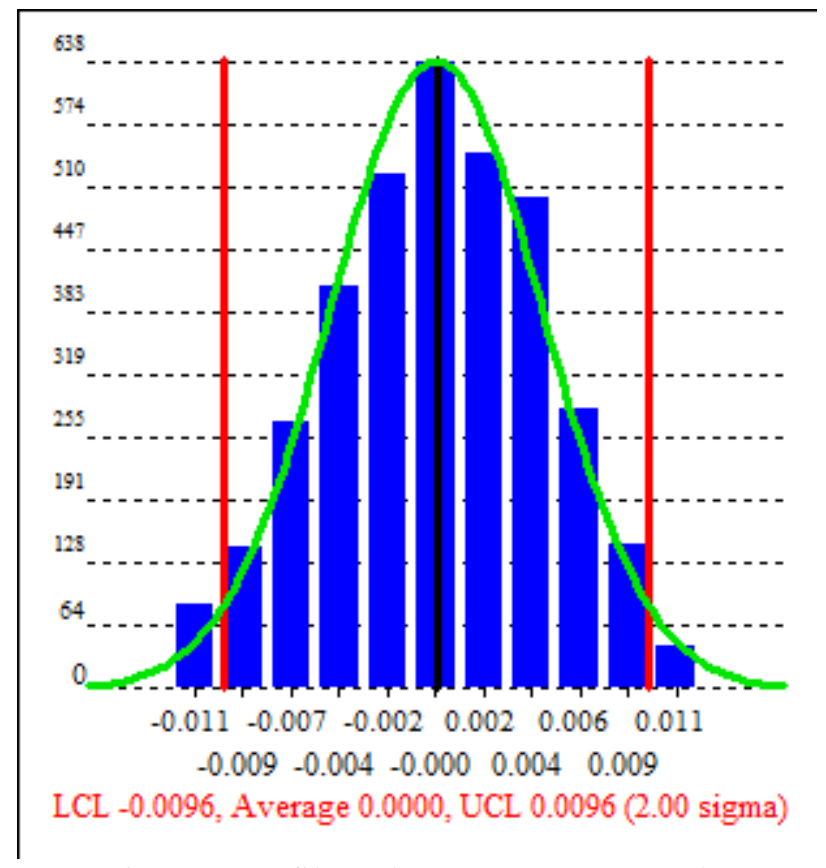

Figure 4. Histogram for 3983 point group, filtered to $+/-0.012 \mathrm{~mm}$ tolerance, all points out of tolerance are ignored. 
The result obtained with the LR is compared to the results obtained with other methods. Laser tracker method \#1 is tramming, where a retro reflector is touched to the surface of the mirror at different points. Laser tracker method \#2 is finding the average distance using the LT and fiduciary surfaces on the mechanical back surface of the mirror and subtracting the mirror thickness measured during fabrication.

Figure 5 shows the results for the various methods.

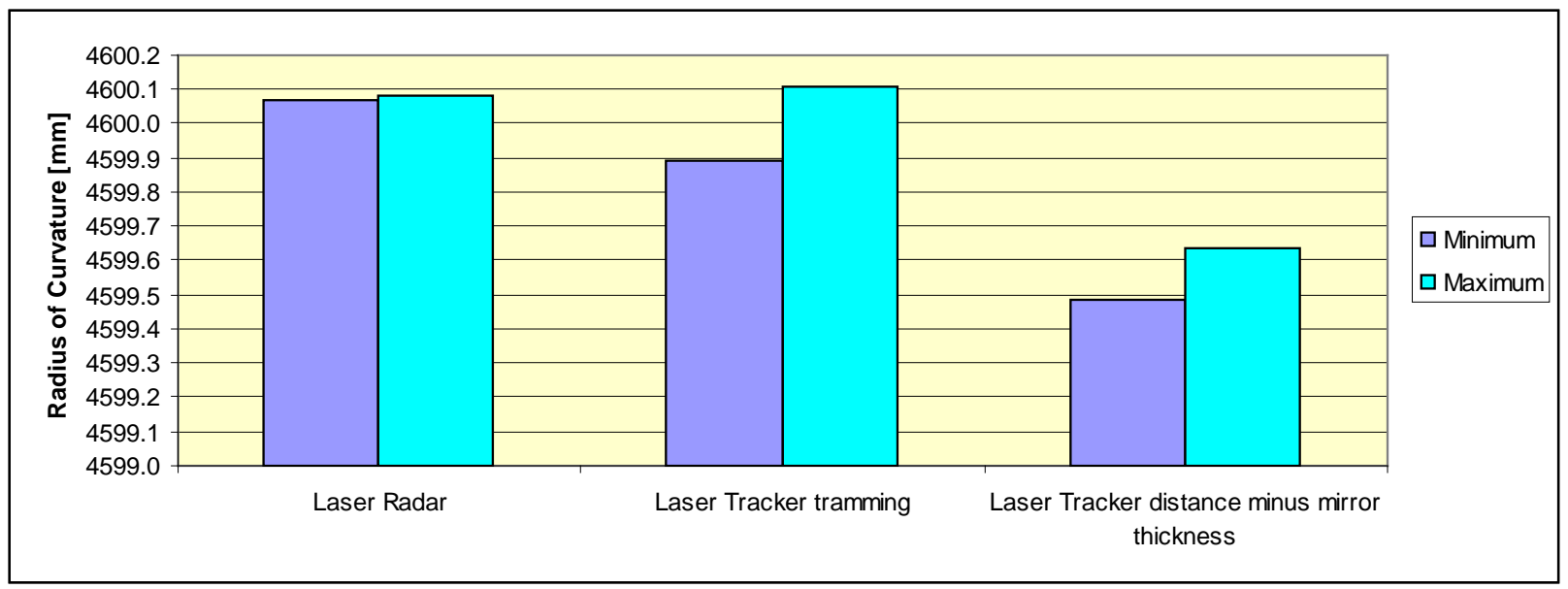

\begin{tabular}{|l|r|r|}
\hline \multicolumn{1}{|c|}{ Method } & \multicolumn{1}{c|}{$\begin{array}{l}\text { Radius of } \\
\text { curvature }\end{array}$} & \multicolumn{1}{c|}{ rms } \\
\hline \multicolumn{1}{|c|}{ Units } & $\mathrm{mm}$ & $\mathrm{mm}$ \\
\hline Laser Radar & 4600.075 & $+/-0.005$ \\
\hline Laser Tracker tramming & 4600.00 & $+/-0.11$ \\
\hline $\begin{array}{l}\text { Laser Tracker distance to mirror back minus mirror } \\
\text { thickness }\end{array}$ & 4599.558 & $+/-0.076$ \\
\hline
\end{tabular}

Figure 5 a and $b$.

a) The maximum and minimum radius of curvature for the different methods.

b) The measured radius of curvature values and associated rms values. 


\section{LASER RADAR POSITIONING SENSITIVITY}

\subsection{Spherical mirrors}

Knowledge about how to position the laser radar for radius of curvature test of a large mirror is important in order to plan and conduct the test. As a best practice, positioning close to the Center of Curvature should be attempted for spherical mirrors. The proximity to the $\mathrm{CoC}$ is driven by the capture range of the camera that collects the return signal from the target.

The LR has a high dynamic range, and is sensitive to a large range of return signals. Non-polished mirrors pose less challenge with respect to positioning the LR than specular surfaces. Because the surface of a non-polished mirror is somewhat diffuse, the probability of rays from each point hitting the detector is much larger, and this makes finding an optimal position for placing the LR less challenging. For a polished, specular surface, such as a finished mirror, which produces returns that follow the law of reflection, care has to be taken to measure the mirror from a spot which allows the return from the specular surface to reach the LR's detector. An uncoated, polished specular mirror can have a reflectivity of about $\sim 0.04$, which poses a separate challenge for LR metrology. Temporary, optical shop coatings can be applied to such a mirror to enable LR measurement. Future LR technology development should make this type of post-polished, but pre-coating surface directly measurable [5].

When positioning the LR for measurement it should be noted that a compensation for the distance between the exit aperture of the LR exit/entrance aperture and the fold mirror, about $100 \mathrm{~mm}$, should be taken into account. To place the LR's aperture at the Center of Curvature (CoC), the instrument base should physically be placed $\sim \mathrm{CoC}-100 \mathrm{~mm}$ away from the mirror vertex. However, there is a tolerance in the placement, which lessens the requirements on how close to the center of curvature the position has to be. If no auxiliary metrology equipment is available to help determine the center of curvature, or it 'real estate' limitations in the test set-up are encountered, measurement of the radius of curvature with the LR is still possible, within certain limits, and iterative alignment can be performed.

The maximum lateral displacement from the optical axis is possible, when the LR is positioned directly at a distance from the mirror equal to the radius of curvature. Here, the LR can theoretically be placed up to $+/$ - half the aperture size away from the optical axis, in the direction orthogonal to the beam line. In the case of these measurements, this number is $+/-20 \mathrm{~mm}$.

For placement uncertainty along the optical axis, the theoretical maximum allowable displacement is found to be $\Delta \mathrm{d}$ = numerical aperture $\mathrm{x}$ detector diameter/2.

As examples, calculations of the theoretical positioning limits for the $\mathrm{R}=4600 \mathrm{~mm}$ OSIM PM are shown in Figure 6. 


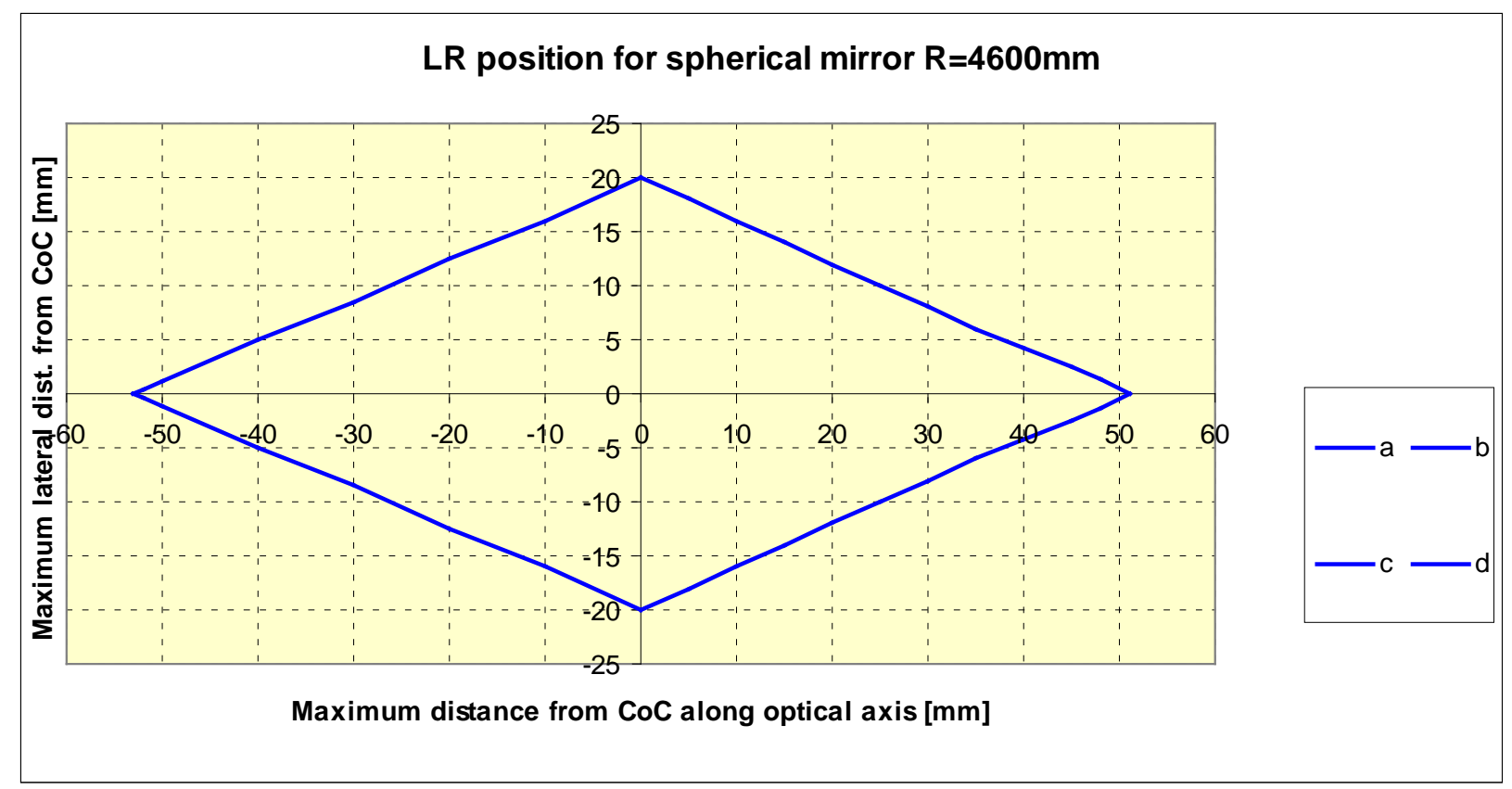

Figure 6. Theoretical limitation on positioning of a LR for measurement of spherical mirror, using simple arguments. The $\mathrm{x}$-axis denotes the displacement from $\mathrm{CoC}$ along the optical axis, the y-axis denotes the lateral displacement from the optical axis.

Since the emitted IR beam is not top-hat shaped, but tapers off at the edges, the signal decreases towards the boundaries of the area defined. How much of this defined area can actually be used for positioning is also dependent on the reflectivity of the surface. The lower the reflectivity, the smaller the area around the CoC can be used.

\subsection{Aspheric mirrors}

Many modern, large, concave mirrors are designed as aspherics. This traditionally poses challenges for testing and fabrication, as compared to similar spherical mirrors. Positioning of the LR for measurement of aspheric mirrors requires higher accuracy in general. For a spherical mirror, from the center of curvature, the distance to each point of the mirror is the same, and at each point of the spherical mirror, the ray will be perpendicular to the local mirror surface, and the light reflected from the mirror will approximately retrace its path and enter the detector in the same way as it emerged from it. For an aspheric mirror, no one location on the mirror surface deviate from lies in the same distance from all points on the mirror.

In order to investigate the positioning tolerance for LR measurement of aspheric mirrors along the optical axis, a model in CodeV is developed. (Corresponding results are obtained using simple ray trace calculations in a spreadsheet. The CodeV model has the advantage of being more flexible). The LR is able to focus its $1550 \mathrm{~nm}$ measurement beam on any object which lies in a distance of $\sim 1 \mathrm{~m}$ to $\sim 24 \mathrm{~m}$ from the radar. (Another LR model is capable of measuring at distances of up to $60 \mathrm{~m}$ ). The focus is set at the beginning of a measurement. In the CodeV model, a simple plano-convex singlet lens with a $40 \mathrm{~mm}$ circular aperture is used, and its focal lengthis adjusted for each measurement distance case. Since the detection system in the LR is not an imaging system, the exact prescription of the lens does not need to be known in order to use the model to predict the optimal position(s) of the LR for a specific aspheric mirror. The fold mirror is moved by the azimuth and elevation encoders and is used to steer the measurement beam towards the object under test is positioned about $100 \mathrm{~mm}$ from the lens. A scanning system in Code $\mathrm{V}$ is used to model this. With the fold mirror at 45 degrees, and the LR positioned on the optical axis of 
the mirror, the apex of the object under test is illuminated. Changing the azimuth angle (x) tilts the mirror to either left or right side. Changing the elevation angle (y) tilts the mirror in or out of the page (Figure 7).

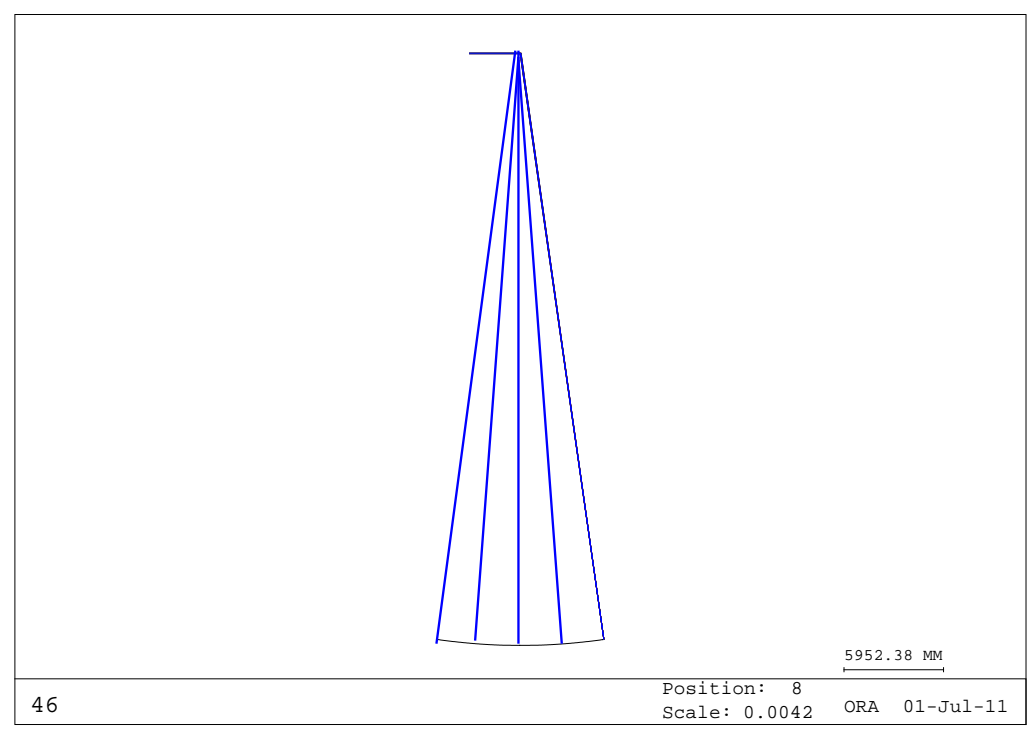

Figure 7. Layout from a Code V model showing 5 different positions of the scan fold mirror. The LR optical train is to the upper left, the mirror under test is at the bottom of the picture.

Varying the scan angle such that the measurement beam hits points from one edge of the mirror to the other, the position of the return beam from the object (mirror) under test is observed. If the return beam does not (partially, at least) fall on the aperture then the signal is not detected. The detector in the LR is a non-imaging detector. It has an acceptance area determined by the $40 \mathrm{~mm}$ aperture, and a dynamic range of $90 \mathrm{~dB}$ which allows it to detect light within the capture area of $1 \mathrm{pW}$. For the cases in which the return beam successfully passes through the aperture on its way back, a measurement is available, depending on the fraction of the aperture covered, the intensity of the return signal, and the reflectivity of the mirror under test. The measured point is stored in the LR, and displayed on the Spatial Analyzer interface screen. The large dynamic range allows detection of signals which only cover a fraction of the non-imaging detector area. The practical limit for detection is a topic for further study, but we assume a threshold of about $10 \%$ of the aperture area is well-within the dynamic range of the detector system (also, note that observing the reflection loss from the fold mirror should nominally be considered as well).

The CodeV model is used to determine LR positioning for measurement of some realistic large mirror examples shown in Table 1.

\begin{tabular}{|l|l|l|l|l|}
\hline $\begin{array}{l}\text { Primary } \\
\text { Mirror }\end{array}$ & Aspheric Type & Diameter & $\begin{array}{l}\text { Radius of } \\
\text { Curvature }\end{array}$ & $\begin{array}{l}\text { Conic } \\
\text { к }\end{array}$ \\
\hline Resembles & & $\mathrm{mm}$ & $\mathrm{mm}$ & \\
\hline 'JWST' & Prolate Ellipse & 6,500 & 15,900 & -0.99666 \\
\hline 'HST' & Hyperbola & 2,400 & 21,500 & -1.0139 \\
\hline 'Keck' & Hyperbola & 10,000 & 35,000 & -1.00368 \\
\hline
\end{tabular}

Table 1. Examples of primary mirrors used in LR positioning study. Primary mirror prescriptions are publicly available. 
The sensitivity towards positioning is determined by the prescription of the mirror, its physical size, numerical aperture, reflectivity, and LR detector size. The model can be used to study this for various mirrors. For the same radius of curvature and numerical aperture, a mirror under test with a conic constant $\kappa$ of $-1<\kappa<0$, a prolate ellipse, will be more 'open' than the corresponding sphere, and therefore the point at which the measurement beam will be near-perpendicular to the mirror surface, ensuring a sufficient return to the LR detector, will be lying further away from the center of curvature point for the mirror. This is also the case for parabolas with $(\kappa=-1)$ and hyperbolic mirrors $(\kappa<-1)$, which shapes are even more open than the corresponding sphere.

Figure 8 shows the fraction of return signal detected as a function of the relative position along the primary mirror radius (where ' 0 ' represents the center of the mirror, and ' 1 ' represents the edge of the mirror) for various positions of the LR along the optical axis. It is seen that for some mirrors, it is advantageous to move the LR away from the Center of Curvature.

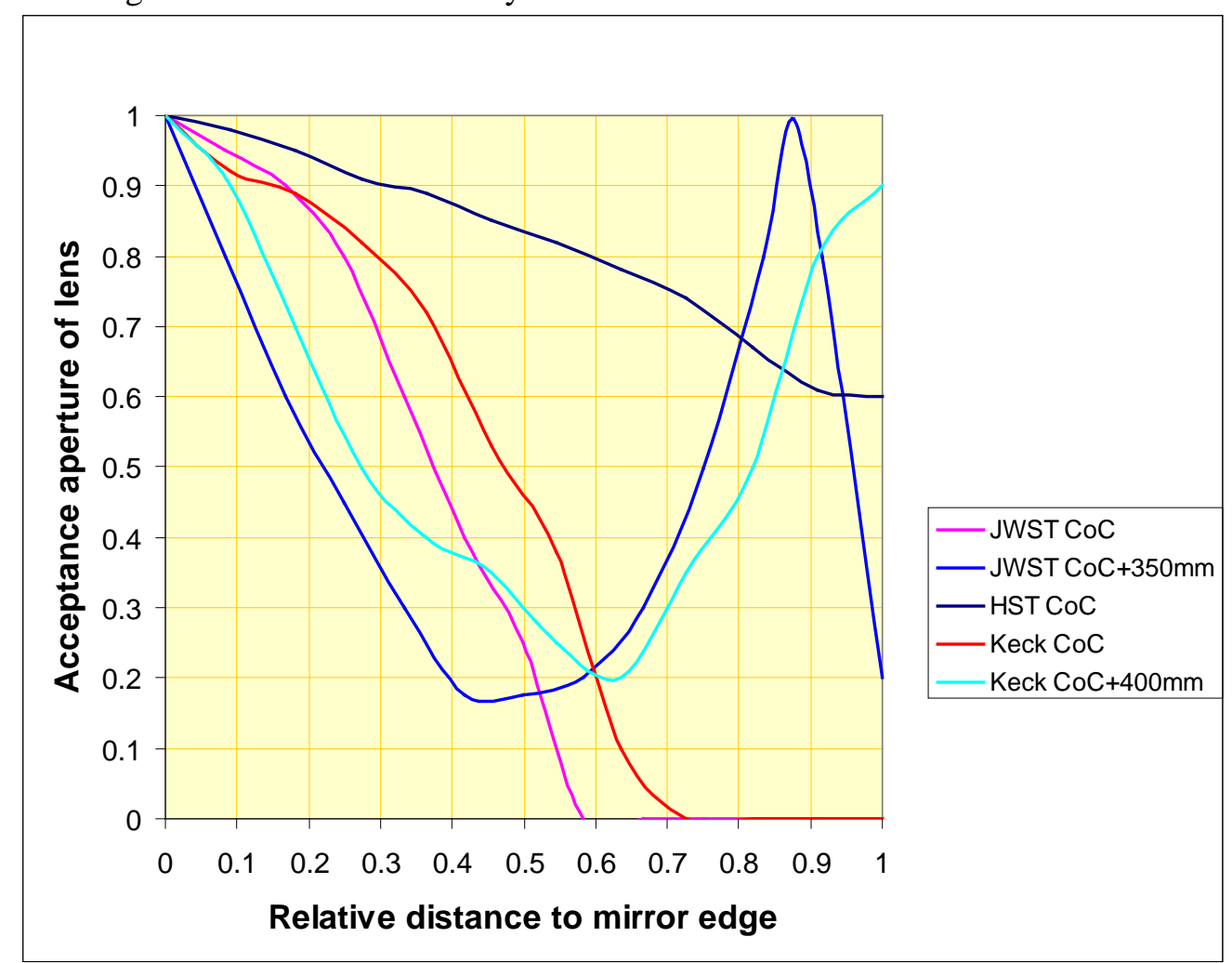

Figure 8. Return signal on $40 \mathrm{~mm}$ aperture as a function of the relative position along the primary mirror radius ( 0 is center of the mirror, 1 is edge of the mirror) for various positions of the LR instrument base along the optical axis.

It is seen from Figure 8 that if the LR is placed at the center of curvature of a particular mirror, only the HST-like mirror would be fully measurable. For the JWST- and Keck-like mirrors, only points around the center, and 6/10 to $7 / 10$ of the way out to the rim are covered. The further back the LR is moved, points closer towards the edge of the mirror are more measurable. In order to measure the outer rim of one of the JWST-like example mirror, the LR should be further from the center of curvature, and in order to measure the parts of the mirror between the center and the rim, the LR should be positioned somewhere in between the $\mathrm{CoC}$ and the outer position. Moving the LR instrument base to a position of about $250 \mathrm{~mm}$ behind the center of curvature, it is possible to balance the error in the position where the return signal hits the detector, so the mirror can be measured from this position with a minimal fraction of the reflected signal reaching the detector from all parts of the mirror. For the Keck-like mirror, moving the LR instrument 
base about $300 \mathrm{~mm}$ behind the center of curvature should produce some fraction of light back from every part of the mirror.

Should there be a mirror for which no one position would produce adequate return signal for a measurement - because of its prescription combined with the reflectance of its surface - it is possible to measure the mirror by performing scans from different positions, and stitch the scans together via post processing of data. Performing more than one scan will inevitably take more time, although it is possible to program the LR to scan in designated areas using pre-defined boundary points. This dual test would in practice be done by positioning the radar on the translator along the optical axis, scan the mirror from the first position, then move the radar, and perform the mirror scan again. Alternatively, one could position fixed fold mirrors in strategic locations to enable a full mirror scan, with over-lapping regions or other tiepoint features, for a fixed LR position. The points measured in common between the two parts of the test will be used to stitch the data to obtain a full mirror measurement, at somewhat increased measurement uncertainty.

The LR is more sensitive to positioning off the optical axis for aspheric mirrors than for spherical mirrors. Dependent on the prescription of the mirror, the scan of an aspheric mirror is prone to drop-outs if the LR is moved more than a couple of $\mathrm{mm}$ away from the optical axis. In cases where the LR needs to be in a set-up which includes other instrumentation at or near the center of curvature, e.g. an interferometer, the use of an auxiliary fold mirror assembly of the use of multiple LR stations may be considered.

The Spatial Analyzer ${ }^{3}$ software, from which the LR instrument interface is commonly run, provides for convenient data analysis based on geometrical fit algorithms, but does not currently support aspheric fits, so aspheric analysis is deferred to a separate software package, such as Matlab ${ }^{4}$ or IDL $^{5}$.

When the LR is positioned for scan, its IR measurement beam is focused on the surface under test. The spot size is minimized to approximately its diffraction-limited size on the point at which the LR head is aimed. While measuring a spherical mirror from the center of curvature, the area covered by the measurement spot is ideally the same distance from the instrument, meaning that no considerable error is added due to the finite spot size. However, if not all points on the surface to be tested are in equal distance from the LR, there will be a focus error in the beam, since the focus is not adjusted during the test. This holds for all spherical surfaces where the radar is not at the absolute center of curvature, as well as for all aspheric and flat surfaces.

The (Airy disk) spot size for the studied example mirrors ranges from $0.217 \mathrm{~mm}$ for OSIM mirror to $1.655 \mathrm{~mm}$ for the Keck-like mirror. A potential error in the range measurement can arise if the spot covers an area with points which do all not lie in the same distance form the mirror. The size of this error can be estimated by calculating how much departure from sphere is found in the area covered by the footprint (spot) of the LR measurement beam.

For a JWST-like mirror, the LR spot size is $0.756 \mathrm{~mm}$, and the departure from sphere is maximum 0.002 $\mathrm{mm}$ at the edge of the mirror. This is small compared to the inherent range error in the instrument of $0.175 \mathrm{~mm}$ for a distance of $16 \mathrm{~m}$. For the shortest measurement distance possible with the radar, $1 \mathrm{~m}$, the range error of $0.03 \mathrm{~mm} 2-\sigma$ still dominates. If the beam is not exactly focused at all points being measured, this changes the spot size. The consequence of this is found to be minimal, especially for the system applications with focal lengths of more than $4000 \mathrm{~mm}$, because the change in spot size is relatively small, less than $50 \%$, for a $10 \mathrm{~mm}$ defocus change.

\footnotetext{
${ }^{3}$ New River Kinematics, Inc., Williamsburg, Va.

${ }^{4}$ MathWorks, Inc.

${ }^{5}$ ITT Visual Information Systems, Inc.
} 


\section{SUMMARY}

At its current state of art and with near-future development, the LR is a flexible, portable, reliable metrology tool, the possible use of which has important implications for the optical fabrication and verification/alignment industry. We propose this non-contact, stand-off technology for various stages in the manufacturing process and subsequent test flow: Without removing the part from the fabrication setup, the LR can be used to measure concave optics and provide nearly real-time feedback to figuring/polishing instruments or other optical grinding or polishing setup. After cleaning, coating, and final verification and alignment/installation to a structure, LR allows precision alignment and characterization of the prescription to levels that promise to improve upon the current state of the art. For advanced levels of integration and test, the large-volume metrology technology would allow fast, noncontact measurement of mirror rigid body alignment and prescription (i.e., radius, conic, aperture), with no special targets or references on the optic. This would allow these mirror parameters to be measured with respect to other optics, instruments, or mechanical- and spacecraft-related structures. For segmented telescope apertures, this possibility includes time saving pre-phasing of segments to micron-level, prior to fine-phasing using other, optical methods.

We have described how the LR can be used to provide a reliable, precise measurement of the radius of curvature of large mirrors. This technique can be applied to both spherical and aspheric telescopes, both monolithic and segmented. Furthermore, we have described the importance of positioning the LR correctly for measurement, and how this positioning is dependent on the prescription of the mirror to be measured.

Future work includes testing of other mirrors, for example, aspheric mirrors and off-axis segments of various prescriptions, and more streamlined data reduction for a range of mirror prescriptions, as well as experimental verification/demonstration of LR positioning sensitivity.

Near-future development of the LR provides an improved coherent detection scheme to reduce range measurement error, as well as better angular encoders on the rotation stages for improved pointing accuracy, which should yield a factor of $\sim 10$ reduction in LR measurement uncertainty. Software improvements should enable better sub-spot detection of mirror apertures and other edge-like features.

\section{ACKNOWLEDGMENTS}

We gratefully acknowledge support from NASA via the James Webb Space Telescope project at the Goddard Space Flight Center. 


\section{REFERENCES}

1. R. Ohl, B. Hoffmann Eegholm, M. Casas, B. Frey, M. Dominguez, J. Gill, J. Hayden, P. Morken, K. Redman, V. Roberts, B. Saif, T. Scirpo, 'Trades for Ambient Non-Contact Metrology,' Proc. SPIE 7433, 743306, 2009.

2. C. Zhao, R. Zehnder, and J. Burge, "Measuring the radius of curvature of a spherical mirror with an interferometer and a laser tracker,” Opt. Eng. 44, SPIE Press, Bellingham, 090506-1—3, 2005.

3. J. Burge, P. Su, C. Zhao, and T. Zobrist, "Use of a commercial laser tracker for optical alignment,” Optical System Alignment and Tolerancing, J. Sasian and M. Ruda, eds., Proc. SPIE 6676, SPIE Press, Bellingham, 66760E-1-12, 2007.

4. A. Slotwinski and J. Genova, "Fiber Optic Coupled Coherent Laser Radar Sensing System for Robotic Applications," SPIE Symposium on Sensing and Perception, Cooperative Intelligent Robots in Space II, Proc. SPIE, SPIE Press, Bellingham, 1991.

5. R. Ohl, B. Eegholm, and A. Slotwinski, “ Laser radar metrology for fabrication, alignment, and verification of large mirrors, Proc.SPIE, Optifab, 2011.

6. James Webb Space Telescope http://www.jwst.nasa.gov/about.html

7. Spatial Analyzer Users Manual, New River Kinematics, Williamsburg, v. 1.21.2008, page 150.

8. S. Sandwith and R. Predmore, "Real-time 5-Micron Uncertainty with Laser Tracking Interferometer Systems using Weighted Trilateration,” Proc. SPIE. 


\title{
LIDAR Metrology for Prescription Characterization and Alignment of Large Mirrors
}

\author{
B. Hoffmann Eegholm
}

Sigma Space Corp., Lanham, Md.

Bente.H.Eegholm@nasa.gov, tel. 3012864342

W. Eichhorn

NASA Goddard Space Flight Center, Code 551, Greenbelt, Md.

R. von Handorf

Ball Aerospace Corporation, Boulder, Co.

J. Hayden

Sigma Space Corp., Lanham, Md.

R. Ohl

NASA Goddard Space Flight Center, Code 551, Greenbelt, Md.

G. Wenzel

Qinetic NA 
- LIDAR (laser radar = LR) technology is demonstrated for use in measuring the radius of curvature of large mirrors

- Advantages are

- Faster, lower manpower costs,

- Reduced tooling needs

- Lower risk of hardware damage,

- Non-contact, in-situ with fabrication equipment

- One metrology solution for multiple stages of telescope manufacture and verification,

- Better accuracy

- Introduction to coherent laser radar (LR)

- Proof of concept test using a meter-class spherical mirror and COTS LR

- Comparison to other methods of radius of curvature measurement

- Discussion of applications for spherical as well as aspheric mirrors

- Current progress in the further development of LR technology for large optics applications

- Future work and summary 


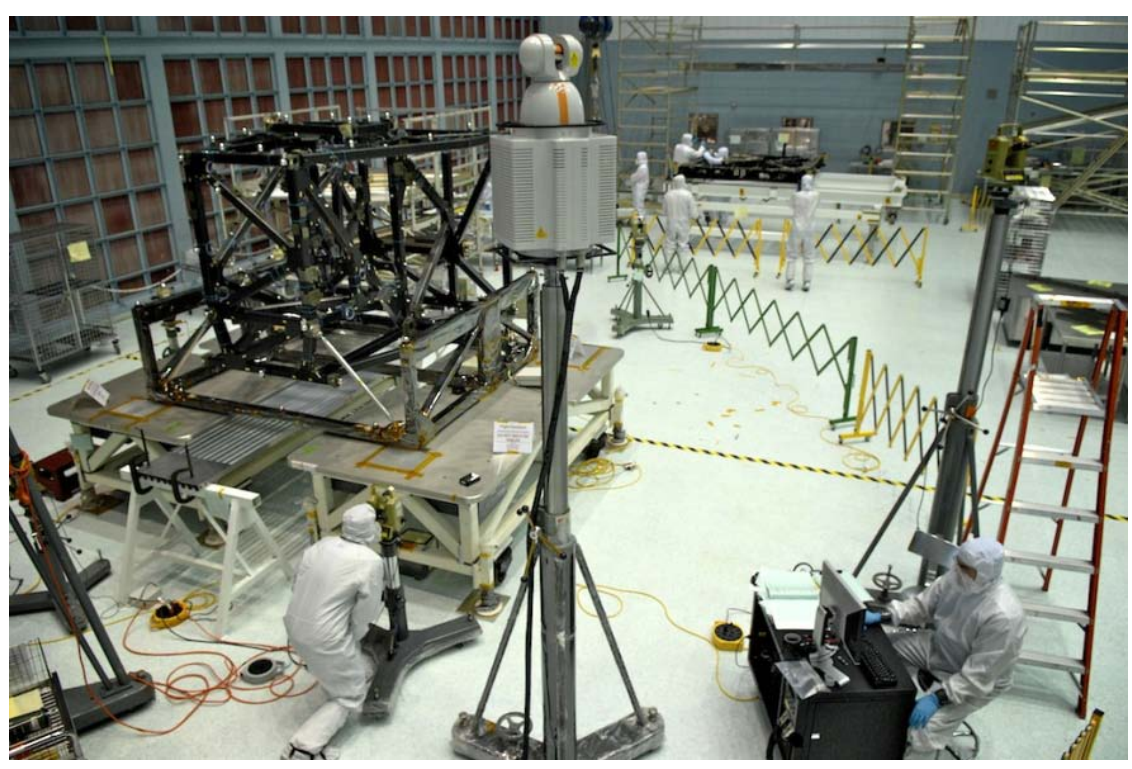

Laser radar and mechanical metrology of part of JWST structure (SSDIF clean room, NASA GSFC)

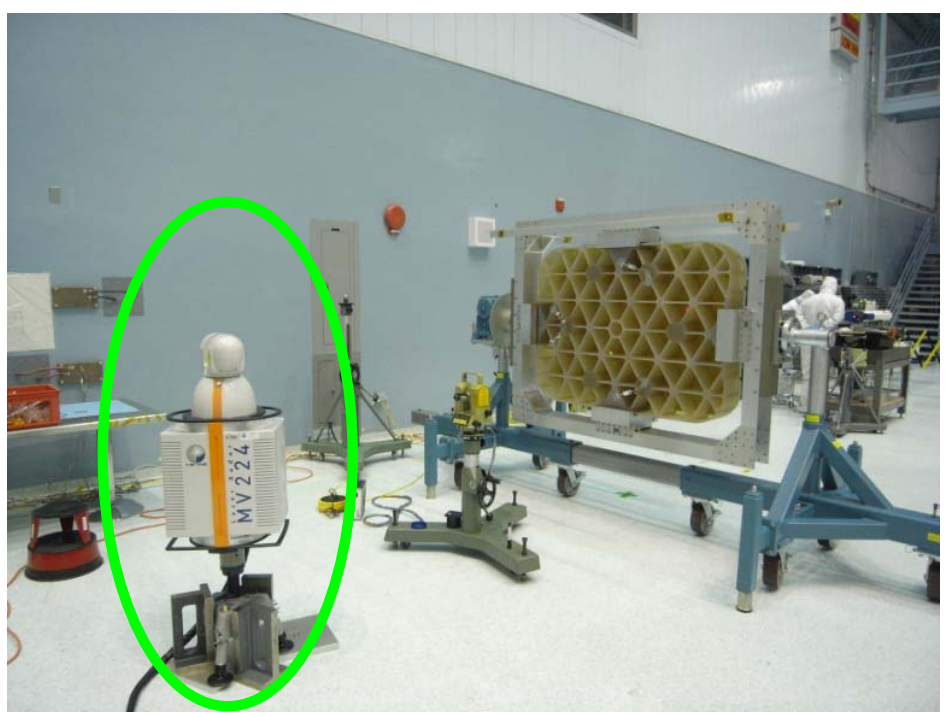

JWST GSE mirror and LR metrology system

(SSDIF clean room, NASA GSFC)

- Application of LIDAR ("laser radar," LR) technology to aid the measurement of the prescription and alignment of large mirrors

- Prescription: Radius, conic, aperture dimensions, off-axis aperture location/orientation

- Alignment: Six degree of freedom alignment to a mechanical coordinate system, alignment to test set during surface fabrication (grinding, polishing), alignment during system integration, coarse phasing of segmented mirror systems

- Process improvement over CMM- or laser tracker-based metrology/alignment systems 
- Commercial laser radar (LR, LIDAR) devices are used in industrial environments for mechanical metrology of parts, structures, assemblies, etc.

- Similar data product to other, spherical, optical-based measurement systems, such as laser trackers

- LR focuses a class I, chirpped1.55 $\mu \mathrm{m}$ laser on a target at a range between $\sim 1-$ 24m (Nikon MV224 LR; Nikon Metrology, Inc., Manassas, Va.) --- 60m possible

- LR collects return energy from the target and optically mixes it with an internal reference signal to obtain a signal whose frequency is proportional to the time of flight to the target

- Data from a weather station is used to compensate for time of flight variation due to different environmental conditions

- An internal interferometer is used as a standard to convert the frequency measurement to range

- LR has a very large dynamic range (>90dB), enabling measurement of many different surface and target types

- Matte-finish, mechanical surfaces (faint scatter return)

- Mirror-like, specular surfaces (bright return via law of reflection)

- No special target types required

- Inexpensive, specular, tooling balls used for point-like coordinate system references (virtual center is detected)

- Various scan types possible (many points per second)

- Measurement automation is straightforward

- Azimuth stage rotates for $>360$ degree coverage horizontally, while the elevation stage provides about \pm 45 degree vertical pointing about the horizon

- Measurement uncertainty

- Range: $\sim 15 \mu \mathrm{m}$ (1-sigma)

- Axes orthogonal to range direction: $\sim 10 \mu \mathrm{m}$ per $1 \mathrm{~m}$ of range (1-sigma)

- Further improvement possible via SA USMN

- Control software: Spatial Analyzer ${ }^{\mathrm{TM}}$ (SA; New River Kinematics, Inc., Williamsburg, Va.)

- Portability: Easy transport; easy to mount at different gravity orientations

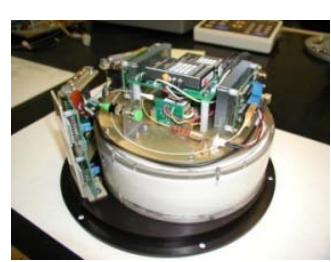

Photonics assembly

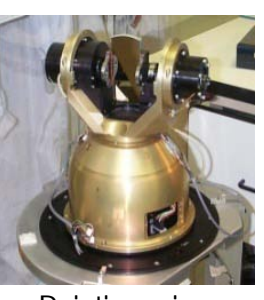

Pointing mirror
assembly

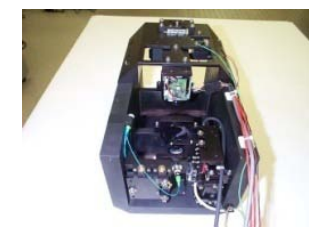

Optics Assembly 西 


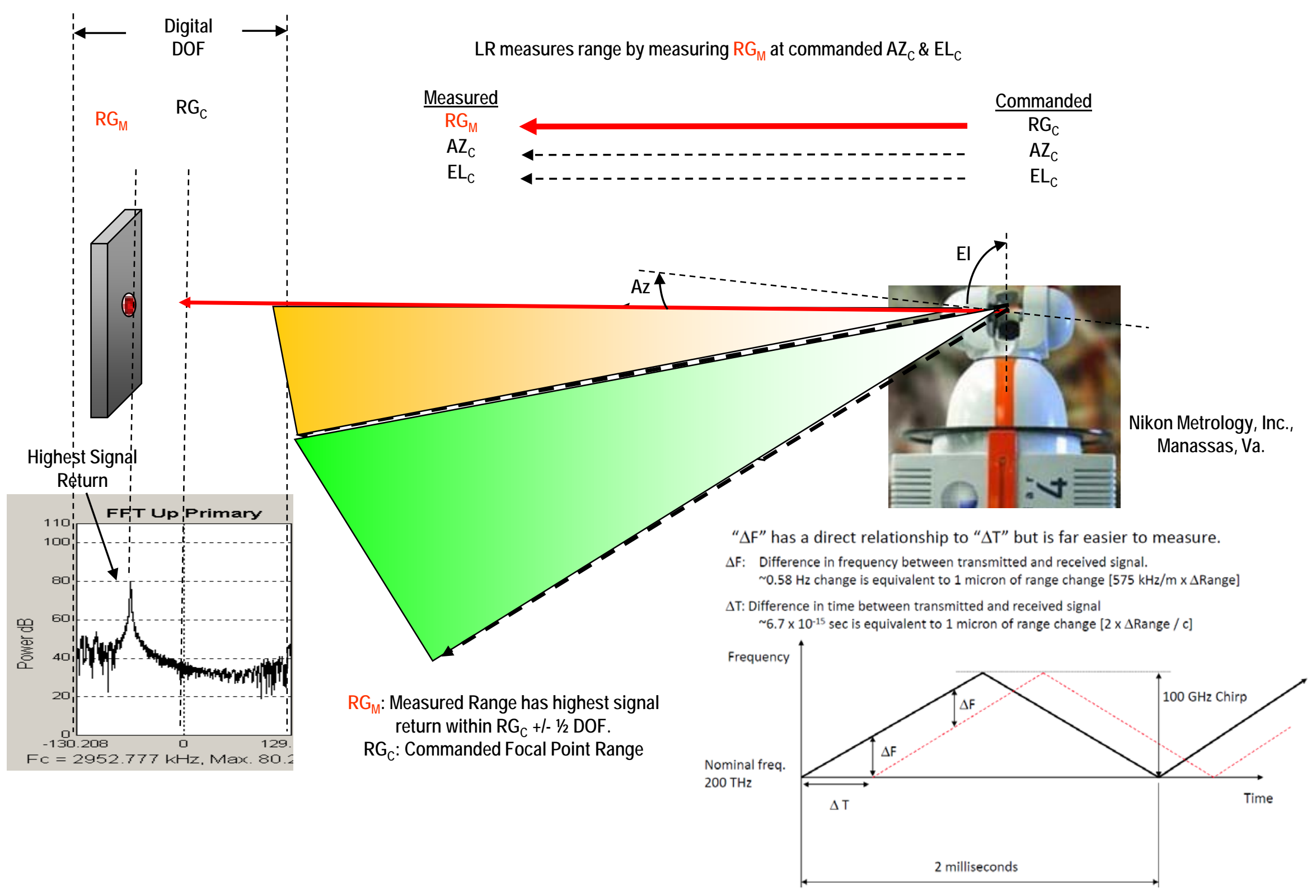




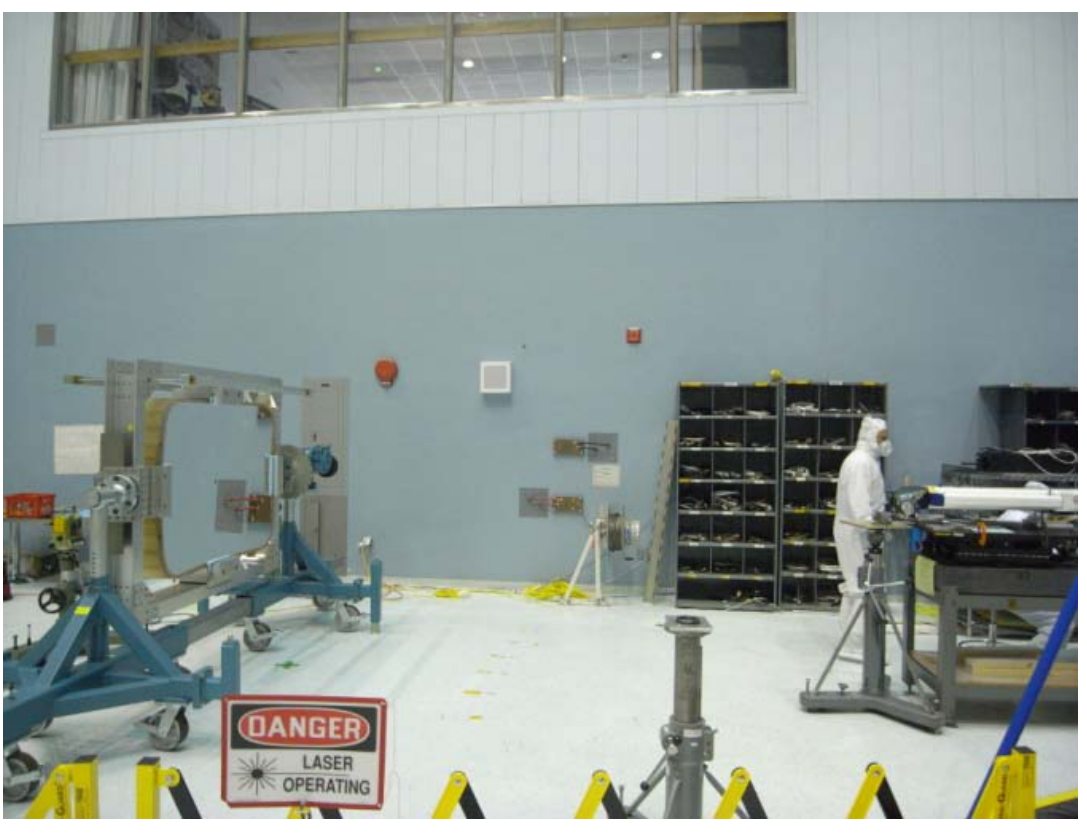

- The $\sim 1.7 \mathrm{~m}$ diameter spherical mirror (left) and the (4D 4020HP) interferometer (right) during figure error testing.

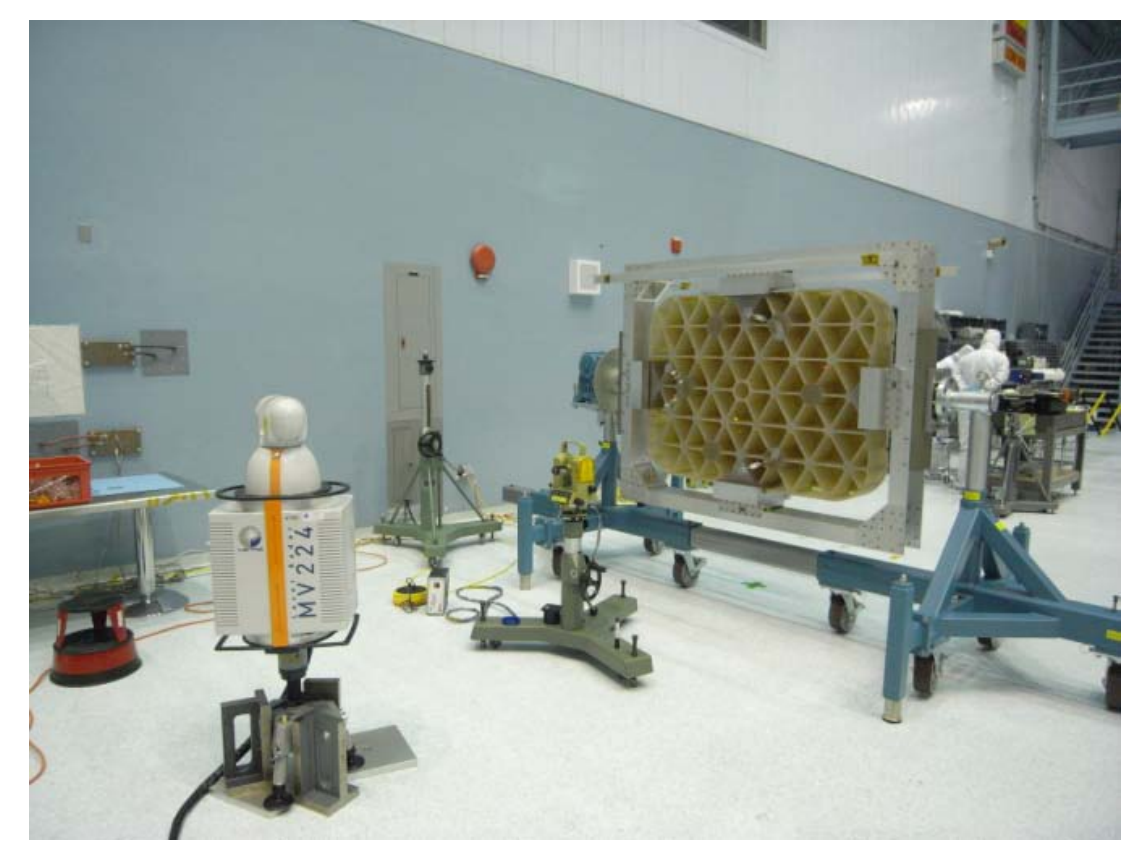

- The LR MV224 (left), theodolite (center), and the mirror (right) during measurement of optomechanical alignment fiducials on the rear surface of the mirror substrate. 
- Purpose: Show that large mirror prescription measurement is feasible using a COTS LR unit by measuring the radius of curvature of a concave, spherical mirror

- Test article:

- Ground support equipment (GSE) mirror for ground test verification of part of the James Webb Space Telescope (JWST) - Large, spherical mirror

- Zerodur ${ }^{\mathrm{TM}}$, class $0,307 \mathrm{~kg}$

- 1.4 wide $x 1 \mathrm{~m}$ hight optical aperture

- Nominal radius of curvature $\mathrm{R}=4600 \mathrm{~mm}$

- Loose requirement on absolute radius: 4594-4606mm

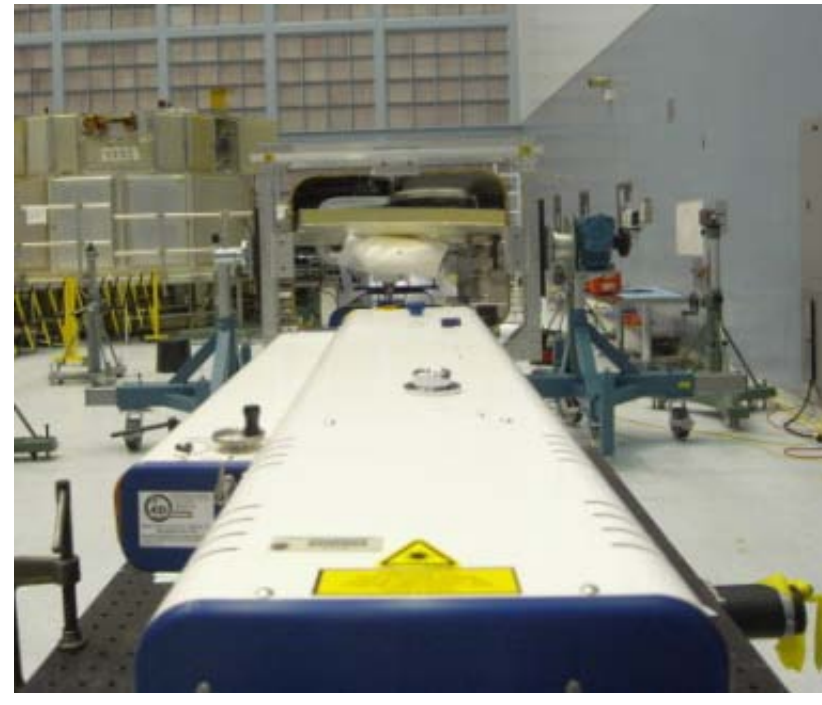

- Figure error requirement (within $380 \mathrm{~mm}$ diameter sub-aperture: $\leq 15 \mathrm{~nm}$ RMS

JWST GSE mirror,

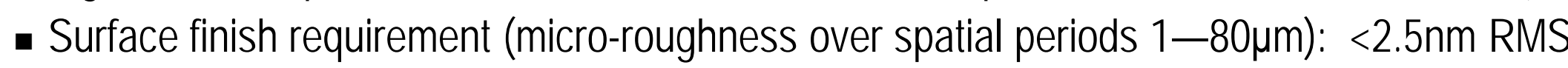

view from interferometer (SSDIF clean room,

- Scratch-dig requirement: 80-50

- LR metrology scan, $\sim 1$ point per $3.5 \mathrm{~cm} 2$ (3983 points)

- Laser radar (LR) result: $4600.075 \mathrm{~mm}$ with RMS deviation from spherical fit of $0.005 \mathrm{~mm}$

- Laser tracker result (mirror vendor reported measurement): $4600+1-0.11 \mathrm{~mm}$ 
Concave mirror under test

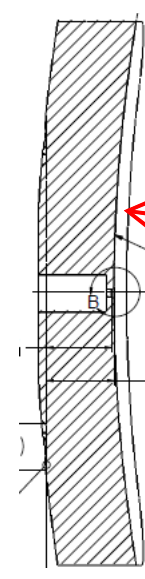

Interferometer test beam

LR beam focused on surface of mirror and specular return (overfills mirror aperture)

Center of curvature of mirror and temporary location of tooling ball target (dashes) used for alignment

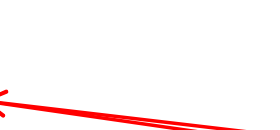
of the interferometer

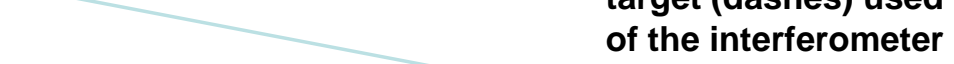

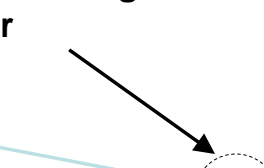

Transmission

sphere

(diverger)

Interferometer instrument located with focus of diverger at center of curvature

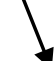

$\downarrow$
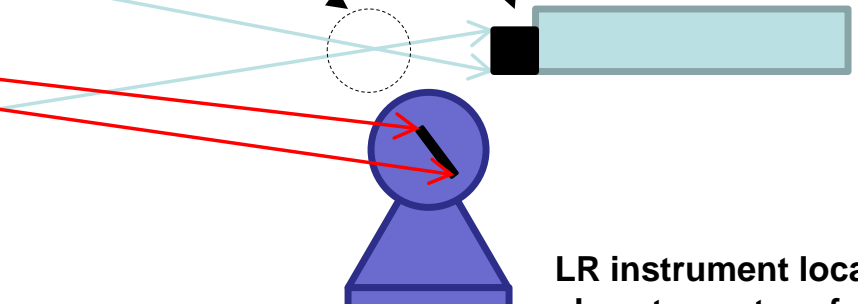

LR instrument located close to center of curvature

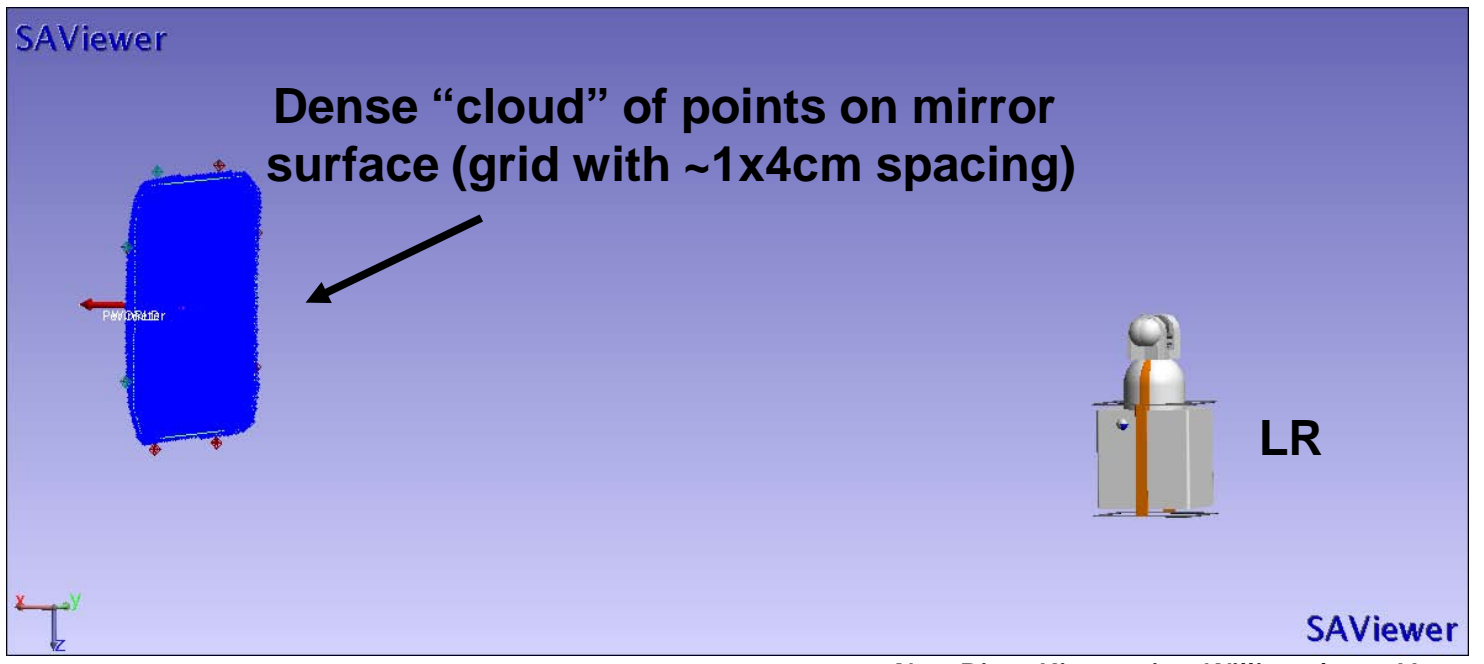

New River Kinematics, Williamsburg, Va.

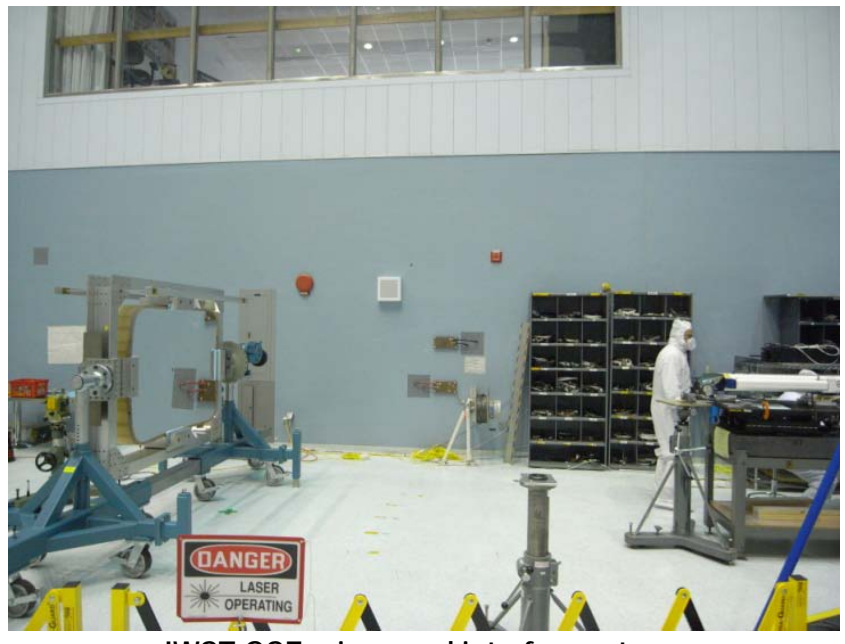

JWST GSE mirror and interferometer (SSDIF clean room, NASA GSFC) 


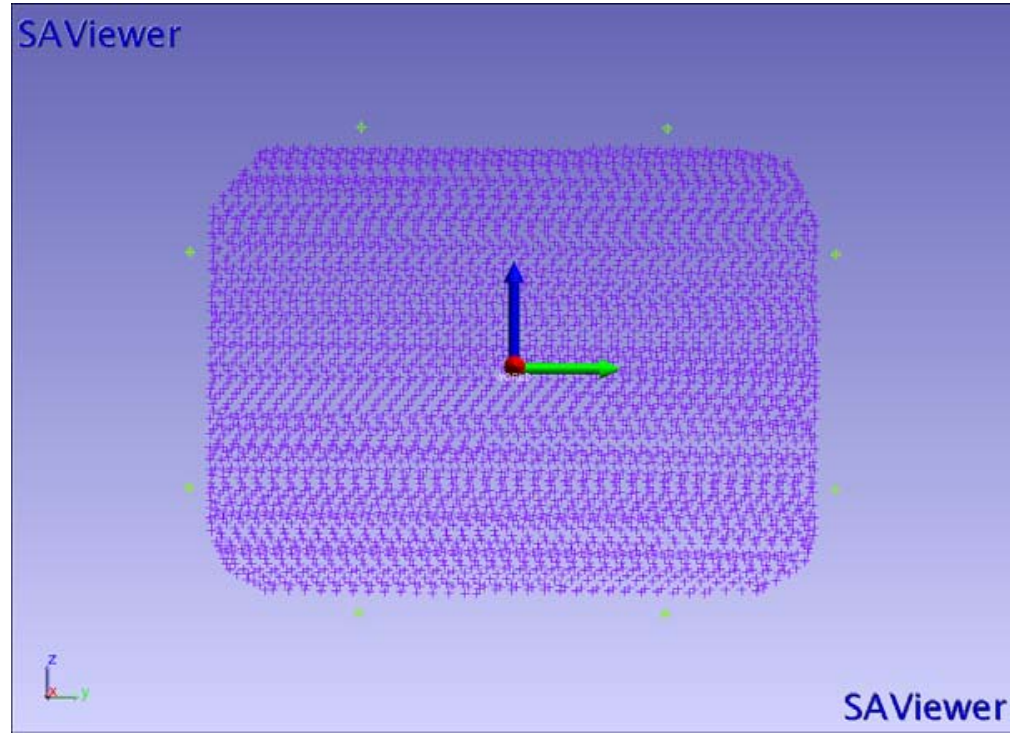

- 3983 points (blue) in the metrology scan

- Green points mark the tooling balls indicating the perimeter of the mirror

- Point density: $\sim 1$ point per $3.5 \mathrm{~cm}^{2}$

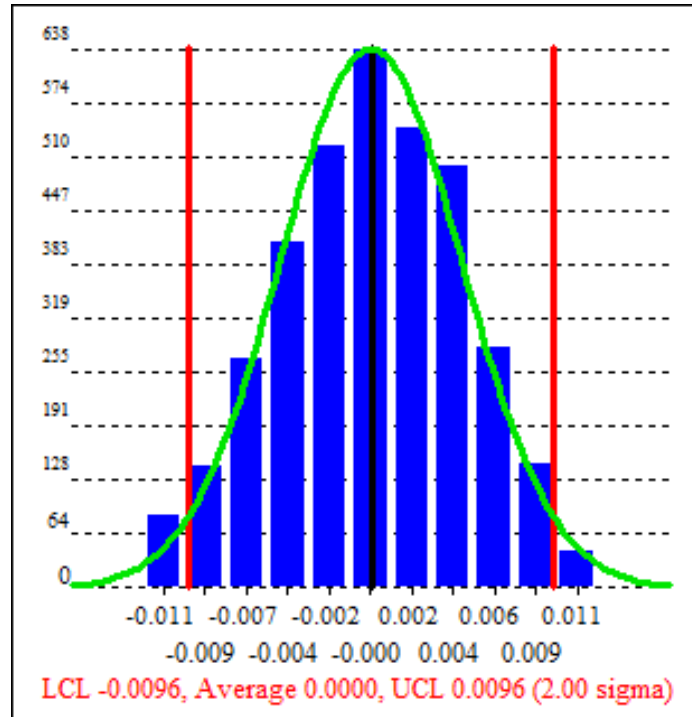

- Histogram for the scan points, filtered to +/- $0.012 \mathrm{~mm}$ tolerance.

- All points out of tolerance are ignored from the least squares fit to a sphere.

- Laser radar (LR) result for radius of curvature: $4600.075 \mathrm{~mm}$ with RMS deviation from spherical fit of $0.005 \mathrm{~mm}$ 


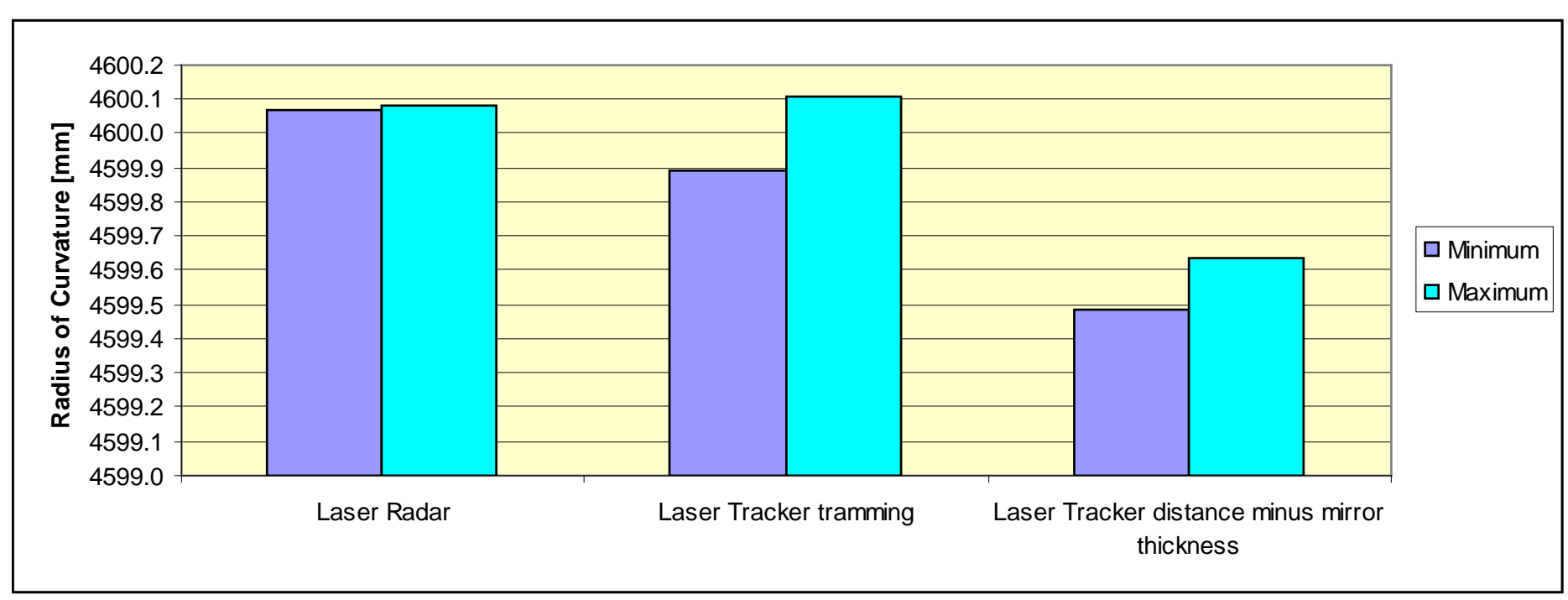

\begin{tabular}{|l|r|c|}
\hline \multicolumn{1}{|c|}{ Method } & \multicolumn{1}{|c|}{$\begin{array}{c}\text { Radius of } \\
\text { curvature }\end{array}$} & $\mathrm{rms}$ \\
\hline \multicolumn{1}{|c|}{ Units } & $\mathrm{mm}$ & $\mathrm{mm}$ \\
\hline Laser Radar & 4600.075 & $+/-0.005$ \\
\hline Laser Tracker tramming & 4600.00 & $+/-0.11$ \\
\hline $\begin{array}{l}\text { Laser Tracker distance to mirror back minus mirror } \\
\text { thickness }\end{array}$ & 4599.558 & $+/-0.076$ \\
\hline & & \\
\hline
\end{tabular}


- Sensitivity study using a CodeV optical model

- Positioning sensitivity of laser radar

- Spherical mirrors

- Aspheric mirrors

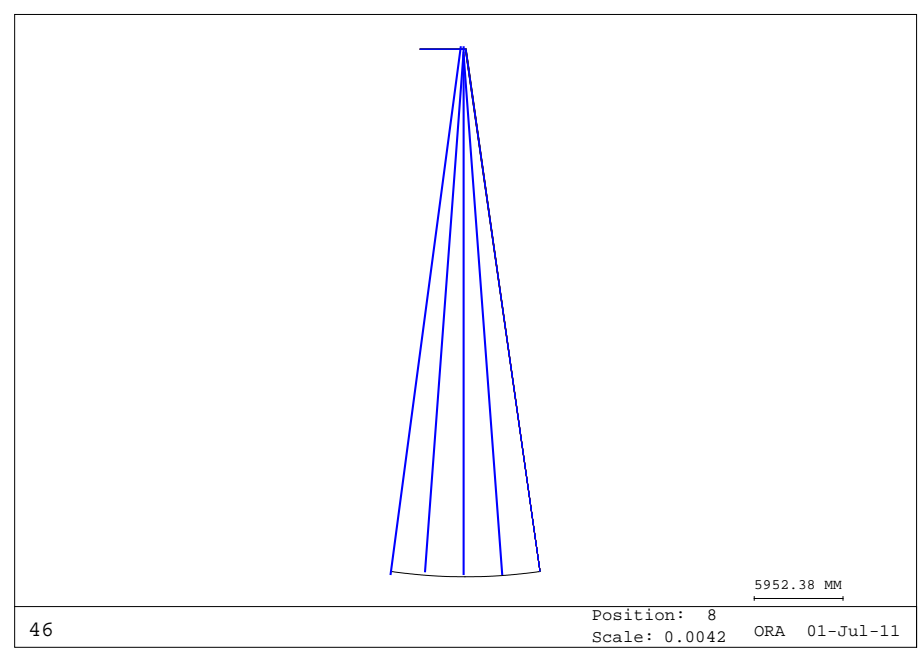

Model incorporates a fold mirror which scans over the optical surface

\begin{tabular}{|l|l|l|l|l|}
\hline $\begin{array}{l}\text { Primary } \\
\text { Mirror }\end{array}$ & $\begin{array}{l}\text { Aspheric } \\
\text { Type }\end{array}$ & $\begin{array}{l}\text { Diamet } \\
\text { er }\end{array}$ & $\begin{array}{l}\text { Radius of } \\
\text { Curvature }\end{array}$ & $\begin{array}{l}\text { Conic } \\
\text { k }\end{array}$ \\
\hline Resembles & & $\mathrm{mm}$ & $\mathrm{mm}$ & \\
\hline 'JWST' & Prolate Ellipse & 6,500 & 15,900 & -0.99666 \\
\hline 'HST' & Hyperbola & 2,400 & 21,500 & -1.0139 \\
\hline 'Keck' & Hyperbola & 10,000 & 35,000 & -1.00368 \\
\hline
\end{tabular}

Examples of primary mirrors used in LR positioning study. Primary mirror prescriptions are publicly available. 


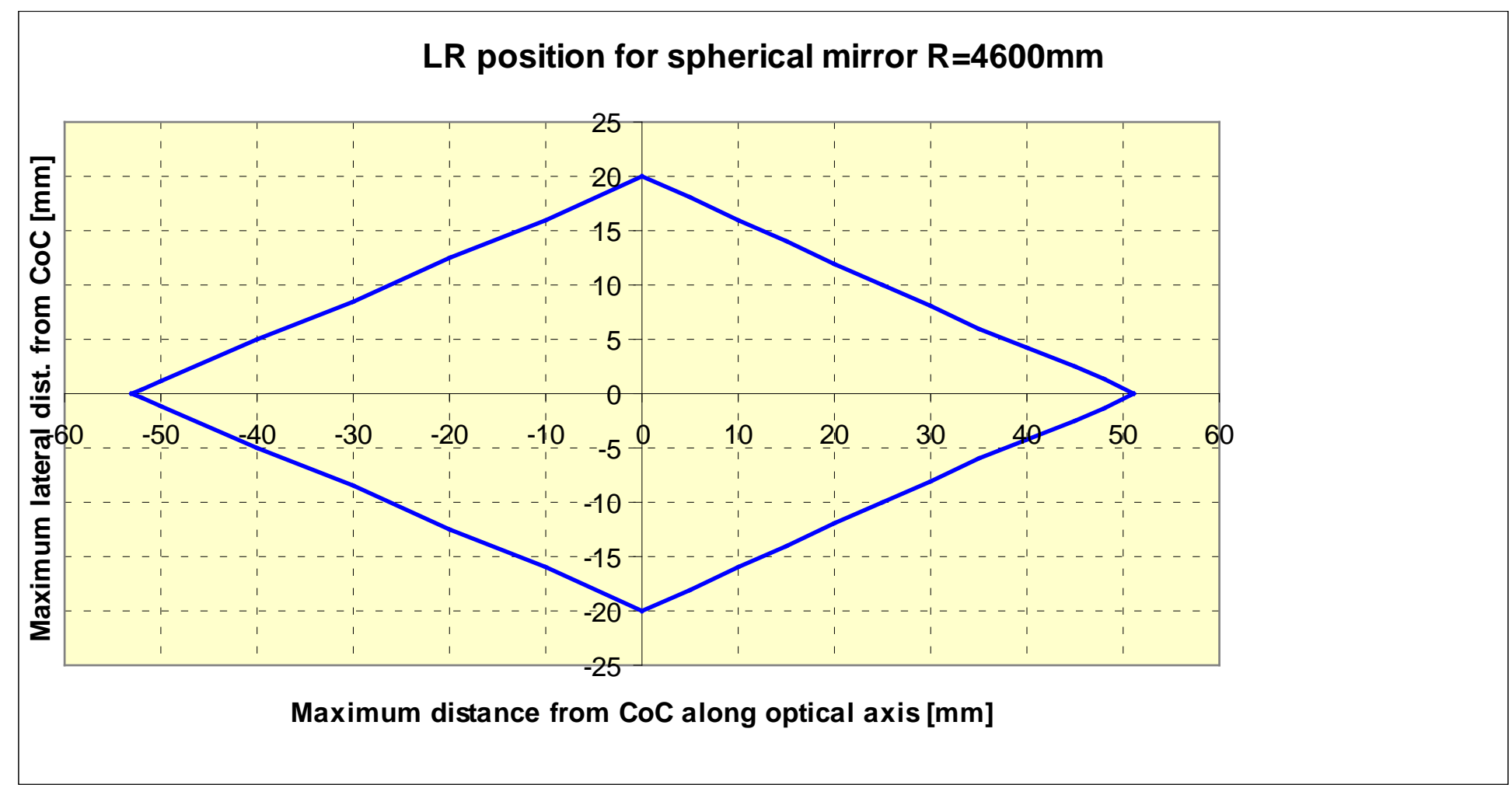

- Theoretical limitation on positioning of a LR for measurement of spherical mirror, using simple arguments.

- The $\mathrm{x}$-axis denotes the displacement from $\mathrm{CoC}$ along the optical axis

- The y-axis denotes the lateral displacement from the optical axis. 


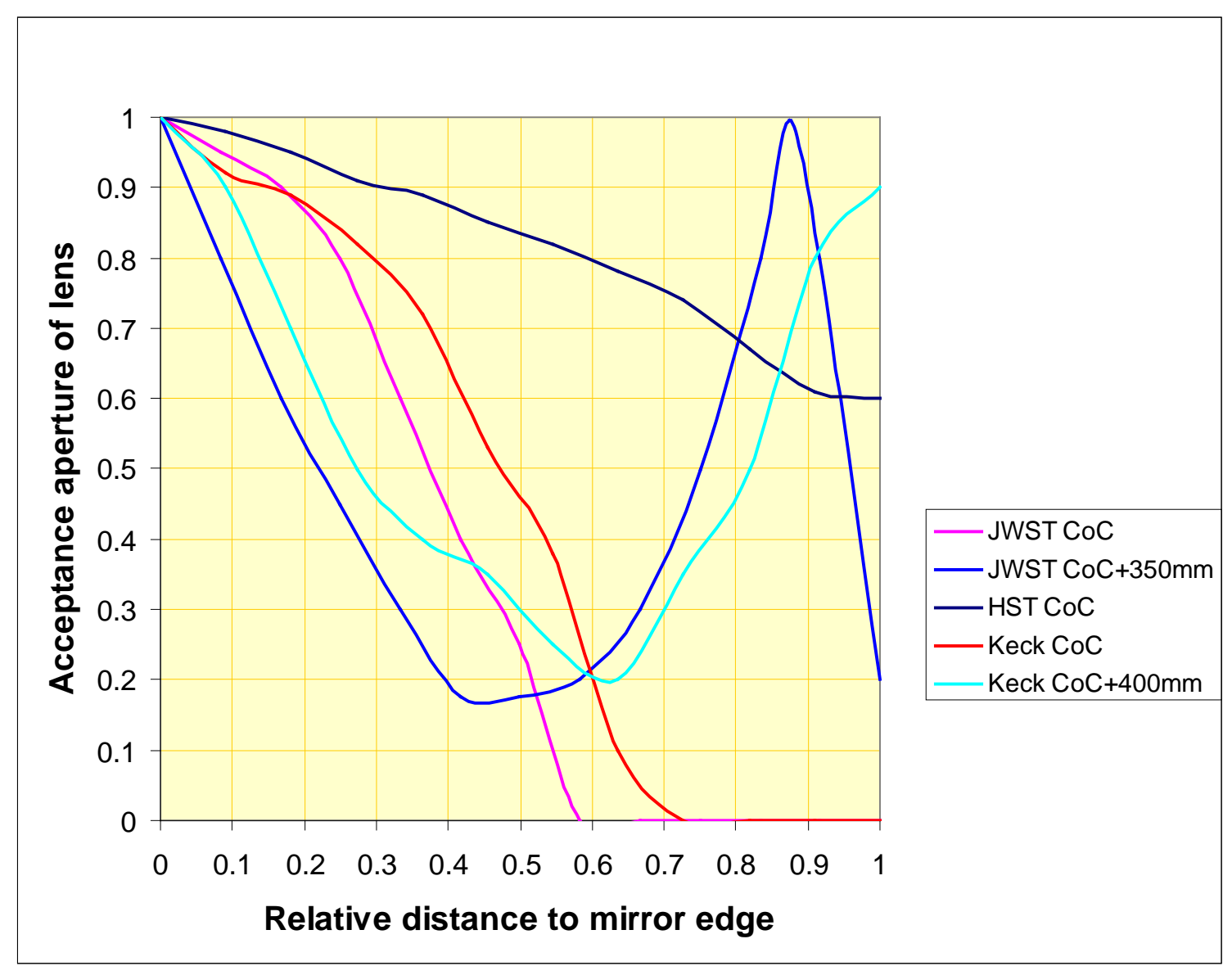

- Return signal on $40 \mathrm{~mm}$ aperture as a function of the relative position along the primary mirror radius ( 0 is center of the mirror, 1 is edge of the mirror) for various positions of the LR instrument base along the optical axis. 


\section{- Results of proof of concept test}

- Fast, non-contact measurement of R (just minutes after brief setup)

- Uncertainty to spherical fit of $\sim 0.005 \mathrm{~mm}$

- Repeatability and absolute uncertainty are expected to be only somewhat larger

- Expected absolute uncertainty is a factor of 10-20 better than mirror vendor's laser tracker-based approach, which required contact with the optical surface

\section{- Post-SBIR development plans:}

- Application to aspheric mirror alignment and testing (e.g., aspheric fit algorithms, characterization of aspheric departure limitations)

- Characterization of uncertainty for measurement of mirror prescription and optical and mechanical edge detection

- Applications to large telescope development and observatory integration and test

- Mirror machining and grinding phases

- Mirror polishing phases

- Mirror final verification for prescription and alignment fiducial calibration

- Mirror assembly/alignment to spacecraft coordinate system

- Ground-based telescope alignment

- Coarse-phasing of future segmented telescopes to within $\sim 1 \mu \mathrm{m}$ 
- We thank T. Slotwinski, PhysicsVision, Inc. for his contributions to this work:

- We gratefully acknowledge support from:

- NASA's James Webb Space Telescope project at the Goddard Space Flight Center

- NASA's Small Business Innovation Research program (contract number NNX10CD71P)
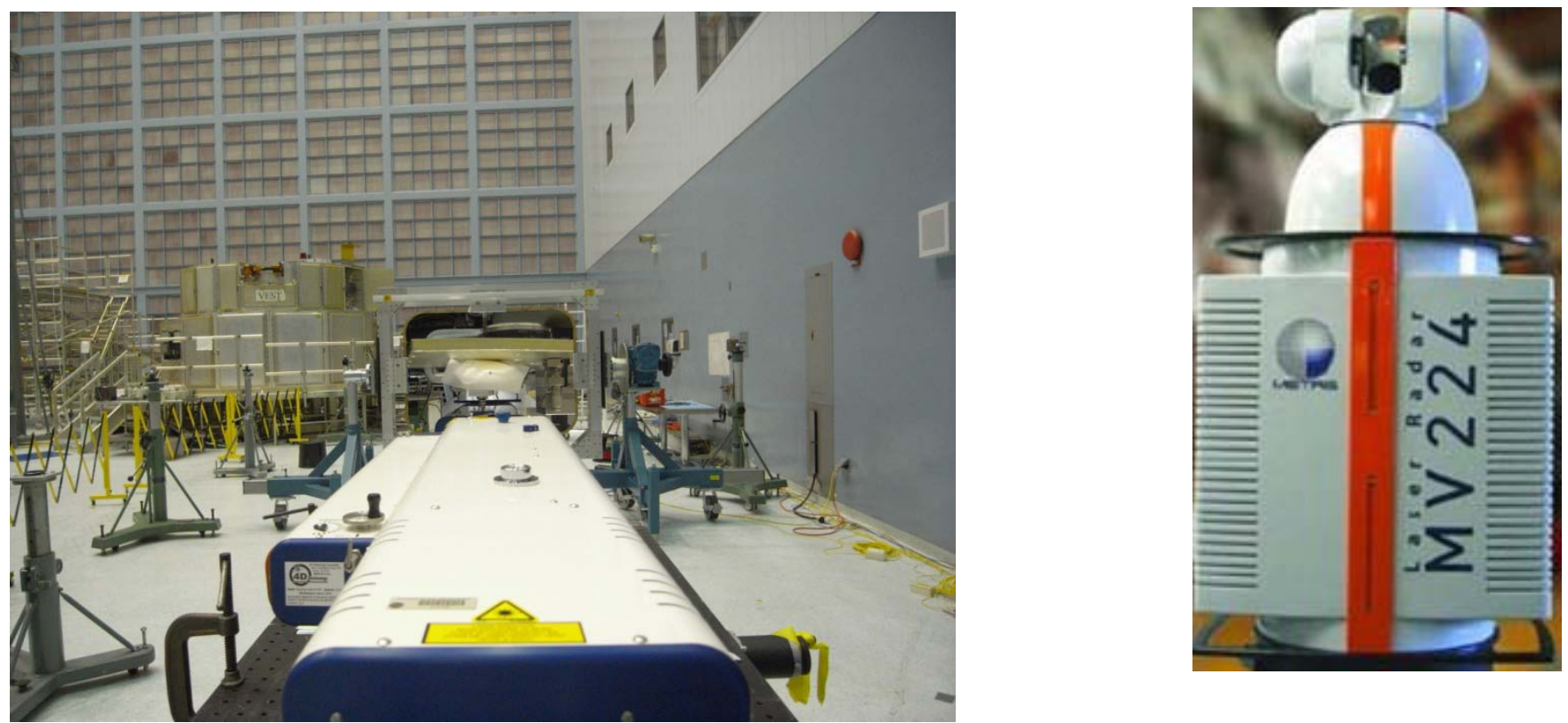
- Additional slides 


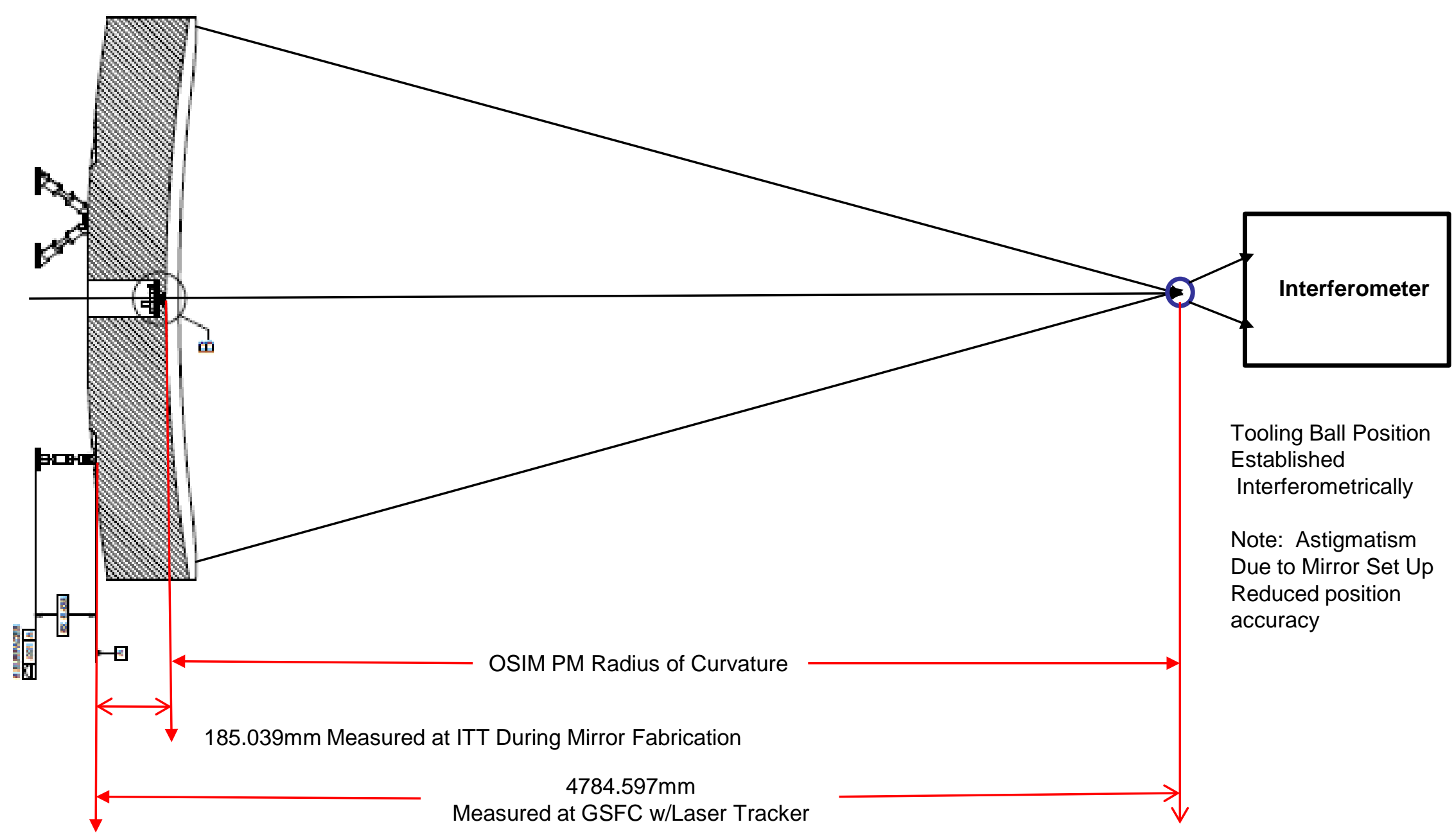


- Added an external, remote weather station to sample laboratory temperature down the beam line for a better optical path correction for the COTS MV224 unit at NASA GSFC

- New type of LR system under development:

- Novel, dual-laser coherent detection scheme reduces both environmental- and scanner-based Doppler ranging error

- Improved encoders for the azimuth and elevation stages

- Measurement uncertainty improvement

- Factor of $\sim 10$ improvement in range uncertainty

- Factor of $\sim 10$ improvement in overall volumetric uncertainty expected

- Increase the capability of the LR's signal processing electronics and software

- Allows user access for analysis of multiple returns per pointing

- Edge detection algorithm improvement (e.g., mirror aperture and mechanical chamfer)

- Detection of uncoated, polished glass mirror surface ( $4 \%$ return)

- Status:

- Phase I SBIR completed testing on a prototype breadboard ranging system which demonstrated a factor of $>10$ improvement in measurement uncertainty

- Phase I SBIR completed engineering studies associated with the design of the final, prototype LR unit

- Phase II SBIR goal is to produce a new prototype LR (Phase II has just started)

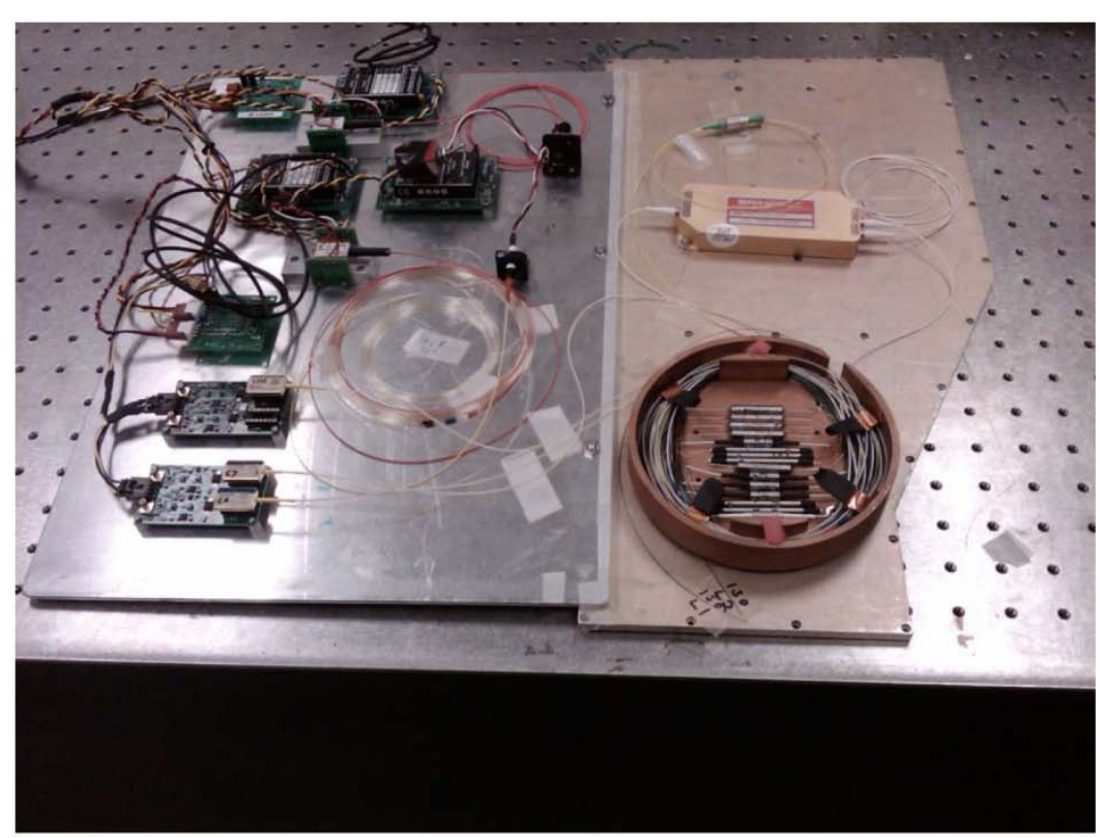

Dual laser radar over prototype hardware

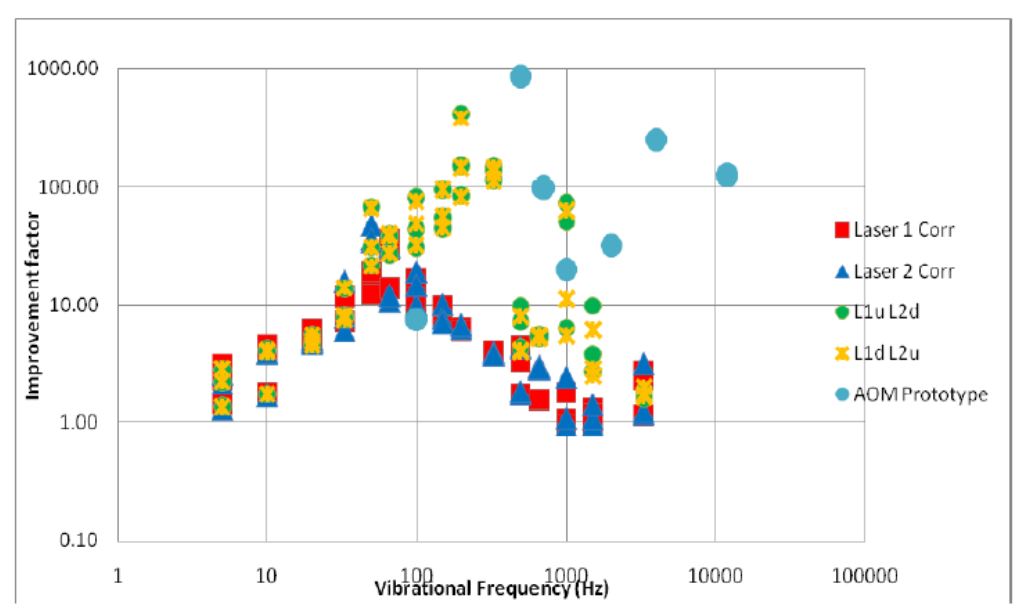

Plot showing Correction summary as a function of oven configuration 